AperTO - Archivio Istituzionale Open Access dell'Università di Torino

\title{
Macrophage PI3Ky drives pancreatic ductal adenocarcinoma progression
}

\section{This is the author's manuscript}

Original Citation:

Availability:

This version is available http://hdl.handle.net/2318/1566699

since 2016-11-04T13:40:46Z

Published version:

DOI:10.1158/2159-8290.CD-15-1346

Terms of use:

Open Access

Anyone can freely access the full text of works made available as "Open Access". Works made available under a Creative Commons license can be used according to the terms and conditions of said license. Use of all other works requires consent of the right holder (author or publisher) if not exempted from copyright protection by the applicable law. 


\section{(3) \\ UNIVERSITÀ DEGLI STUDI DI TORINO}

This is an author version of the contribution published on:

Questa è la versione dell'autore dell'opera:

Cancer Discovery 2016. doi: 10.1158/2159-8290.

ovvero [Kaneda MM., May 13, AACR Journal, 2016, pagg CD15-1346]

The definitive version is available at:

La versione definitiva è disponibile alla URL:

http://cancerdiscovery.aacrjournals.org/content/early/2016/05/13/2159-8290.CD-

15-1346.abstract 


\section{Macrophage PI3K $\gamma$ drives pancreatic ductal adenocarcinoma progression}

Megan M. Kaneda ${ }^{1}$, Paola Cappello ${ }^{2,3}$, Abraham V. Nguyen ${ }^{1}$, Natacha Ralainirina ${ }^{1}$, Chanae R. Hardamon $^{1}$, Philippe Foubert ${ }^{1}$, Michael C. Schmid ${ }^{1}$, Ping Sun ${ }^{1,5}$, Evangeline Mose ${ }^{1}$, Michael Bouvet $^{1,6}$, Andrew M. Lowy ${ }^{1,6}$, Mark A. Valasek ${ }^{1,7}$, Roman Sasik ${ }^{8}$, Francesco Novelli ${ }^{2,3,9}$, Emilio Hirsch $^{3,4,9}$ and Judith A. Varner ${ }^{1,7,9}$

${ }^{1}$ Moores Cancer Center, University of California, San Diego, La Jolla, CA

${ }^{2}$ Center for Experimental Research and Medical Studies (CeRMS), Azienda Ospedaliera Universitaria Città della Salute e della Scienza di Torino, Turin, Italy

${ }^{3}$ Department of Molecular Biotechnology and Health Sciences, University of Torino, Torino, Italy

${ }^{4}$ Molecular Biotechnology Center, Torino, Italy

${ }^{5}$ Department of Pathology, Mudanjiang Medical University, Mudanjiang, China

${ }^{6}$ Department of Surgery, University of California, San Diego, La Jolla, CA

${ }^{7}$ Department of Pathology, University of California, San Diego, La Jolla, CA

${ }^{8}$ Center for Computational Biology and Bioinformatics, University of California, San Diego, La Jolla, CA

${ }^{9}$ These authors contributed equally; addresses for correspondence:

Judith A. Varner, Ph.D.

Department of Pathology

Moores Cancer Center

University of California, San Diego

3855 Health Sciences Drive

La Jolla, CA 92093-0819

Voice: 858-822-0086

Fax: 858-822-1325

email: jvarner@ucsd.edu

Francesco Novelli, Ph.D.

CeRMS-Lab Tumor Immunology

via Santena 5-10126 Torino, Italy

Voice: +390116336886

Fax: +39011 6336886 
email: franco.noveli@unito.it

Emilio Hirsh, Ph.D.

Molecular Biotechnology Center

via Nizza 62-10126 Torino, Italy

Voice: +390116706425

Fax: +39011

email: emilio.hirsch@unito.it

Running title: PI3Kgamma is a novel therapeutic target in pancreas cancer

Key words: PI3K $\gamma$, PDGF, Arginase, transcription, macrophages, immune suppression, chemotherapy, gemcitabine, pancreatic ductal adenocarcinoma, desmoplasia, metastasis

Conflicts: None 


\section{Abstract}

Pancreatic ductal adenocarcinoma (PDAC) is a devastating disease with a low five-year survival rate, yet new immunotherapeutic modalities may offer hope for this and other intractable cancers. Here we report that inhibitory targeting of PI3K $\gamma$, a key macrophage lipid kinase, stimulates anti-tumor immune responses, leading to improved survival and responsiveness to standard-of-care chemotherapy in animal models of PDAC. PI3K $\gamma$ selectively drives immunosuppressive transcriptional programming in macrophages that inhibits adaptive immune responses and promotes tumor cell invasion and desmoplasia in PDAC. Blockade of PI3K $\gamma$ in PDAC-bearing mice reprograms tumor-associated macrophages to stimulate $\mathrm{CD} 8^{+} \mathrm{T}$ cellmediated tumor suppression and to inhibit tumor cell invasion, metastasis and desmoplasia. These data indicate the central role that macrophage PI $3 \mathrm{~K} \gamma$ plays in PDAC progression and demonstrate that pharmacological inhibition of $\mathrm{PI} 3 \mathrm{~K} \gamma$ represents a new therapeutic modality for this devastating tumor type. 


\section{Significance}

We report here that PI3Kinase gamma $(\mathrm{PI} 3 \mathrm{~K} \gamma)$ regulates macrophage transcriptional

programming leading to $\mathrm{T}$ cell suppression, desmoplasia and metastasis in pancreas adenocarcinoma. Genetic or pharmacological inhibition of PI3K $\gamma$ restores anti-tumor immune responses and improves responsiveness to standard-of-care chemotherapy. PI3K $\gamma$ represents a new therapeutic immune target for pancreas cancer. 


\section{Introduction}

Inflammation plays a major role in cancer immune suppression and progression, perhaps especially in pancreatic cancer (1-4). Ductal adenocarcinoma of the pancreas (PDAC), a devastating disease with one of the poorest five-year survival rates among all cancers, is the third leading cause of cancer deaths in America (5-6). Although 10-15\% of patients are candidates for gross total surgical resection, local recurrence and metastasis are frequent, and the overall 5-year survival rate among patients with pancreatic cancer is only around 7\% (5-6). Since standard therapies have only a modest impact on survival (7-8), novel therapeutic and diagnostic strategies are urgently needed.

Pancreatic carcinomas, like other solid tumors, are characterized by an abundant inflammatory cell infiltrate, including T cells, B cells, mast cells, macrophages and neutrophils, yet these cells produce an ineffective anti-tumor immune response (9-11). Myeloid lineage cells, including macrophages, neutrophils, mast cells and myeloid derived suppressor cells, play important roles in the initiation of pancreatic carcinoma and in establishing an immune suppressive microenvironment that dampens effective anti-tumor $\mathrm{T}$ cell responses in pancreatic carcinomas (9-11). Strategies to reverse immune suppression in PDAC by inhibiting myeloid cell 
trafficking or signal transduction (12-16), by re-activating adaptive immune responses, by vaccination with attenuated intracellular bacteria or DNA plasmids coding for PDAC-associated antigens (16-18), or by the application of checkpoint inhibitors (19-21), have demonstrated that appropriate targeting of the immune system may lead to new and effective treatment strategies for pancreatic cancer. However, continued investigation into the mechanisms by which innate immune cells contribute to PDAC progression will help to refine approaches to improve therapy for this disease.

For this reason, we sought to identify mechanisms regulating the contribution of myeloid cells to immune suppression and progression of PDAC. We report here that the macrophage lipid kinase $\mathrm{PI} 3 \mathrm{~K} \gamma$ promotes immune suppressive polarization in macrophages, leading to immune suppression, tumor progression, metastasis and fibrosis in PDAC. The Class I PI3K lipid kinases phosphorylate PtdIns $(4,5) \mathrm{P} 2$ on the 3' hydroxyl position of the inositol ring to produce PtdIns $(3,4,5) \mathrm{P} 3$, which regulates metabolic and transcriptional pathways during inflammation and cancer (22-23). The Class IA isoforms PI3K $\alpha$ and $\beta$ are widely expressed in endothelial, epithelial and tumor cells, while the Class IA isoform PI3K $\delta$ is primarily expressed in $\mathrm{T}$ and $\mathrm{B}$ lymphocytes and the structurally unique Class IB isoform PI3K $\gamma$ is expressed mainly in myeloid cells where it is the major PI3K isoform (22-23). PI3K $\gamma$ promotes the adhesion and migration of 
granulocytes and monocytes, and mice lacking the PI3K $\gamma$ catalytic subunit, exhibit defects in

neutrophil and monocyte accumulation in inflamed and tumor tissues (24-25). Using orthotopic and genetically engineered mouse models of PDAC, we found that inhibition of PI3K $\gamma$ unexpectedly slowed PDAC tumor growth and enhanced survival and responsiveness to standard-of-care chemotherapy by altering macrophage transcriptional profiles and thereby activating $\mathrm{T}$ cells, indicating that targeting PI3K $\gamma$ therapeutically could provide long-term control of this devastating malignancy.

\section{Results}

\section{PI3K $\gamma$ is a marker of pancreatic ductal adenocarcinoma associated macrophages}

Macrophages play particularly significant roles in PDAC tumor progression, as they can promote tumor initiation, immune suppression and metastasis (9-15). To investigate the role of the macrophage in spontaneous and experimentally induced pancreatic adenocarcinomas, we immunostained pancreata from normal and tumor bearing mice to detect the presence of F4/80+ macrophages. We examined pancreata from normal mice, as well as $L S L-K \operatorname{Ras}^{G 12 D}$; Pdx-1Cre mice (KC mice), which develop extensive PanIns but not invasive carcinoma, LSL-KRas ${ }^{G 12 D /+}$; 
LSL-Trp53 ${ }^{R 172 H /+} ;$ Pdx-1Cre mice (KPC mice), which develop invasive carcinomas and widespread metastases, and orthotopically implanted $L S L-K R a s^{G 12 D /+} ; L S L-T r p 53^{R 172 H /+} P d x-$ 1Cre (LMP) tumors (26-32). While normal pancreata exhibited very few F4/80+ macrophages, pancreata from $\mathrm{KC}$ and $\mathrm{KPC}$ mice exhibited extensive infiltration by macrophages, as did orthotopic LMP tumors (Fig. 1A). Quantification of CD11b+ myeloid cells and F4/80+ macrophages in spontaneous and orthotopic LMP tumors demonstrated that macrophage infiltration increases, while $\mathrm{T}$ cell and $\mathrm{B}$ cell infiltration decreases during tumor development (Fig. 1B and Supplementary Figure S1A-D). We also observed increases in CD68+ macrophage content in invasive pancreatic ductal adenocarcinomas compared to healthy pancreata from patients (Fig. 1C-D), as has been previously reported (26), indicating that increased macrophage infiltration occurs in both murine and human PDAC.

The infiltration of bone marrow derived granulocytes and monocytes into tumors depends in part on the gamma isoform of the Class I PI3K lipid kinases (24-25). Therefore, we asked whether $\mathrm{PI} 3 \mathrm{~K} \gamma$ might promote inflammation associated with tumor progression in PDAC. Immunostaining of clinical biopsies of human invasive pancreatic ductal adenocarcinomas to detect expression of $\mathrm{PI} 3 \mathrm{~K} \gamma$ (green) and $\mathrm{CD} 68+$ macrophages (red) showed that CD68+ macrophages, but not other cells in pancreatic tumors, uniformly express PI3K $\gamma$ (yellow) (Fig. 
1E). Western blotting analyses showed that murine bone marrow derived and tumor associated

macrophages express PI3K $\gamma$, while CD19+ B cells and CD90+ T cells express PI3K $\delta$ and murine

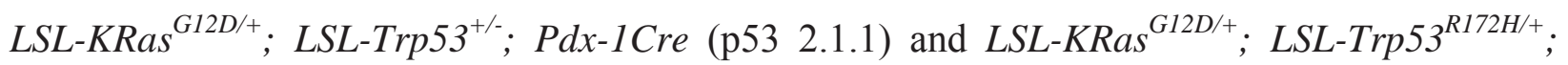

Pdx-1Cre (LMP, K8484, DT6606, or DT4313) pancreatic carcinoma cells express PI3K $\alpha$ but not

PI3K $\gamma$ (Fig. 1F and Supplementary Fig. S2A). These results indicate that PI3K $\gamma$ is a biomarker of

PDAC-associated macrophages that might have a functional role in PDAC inflammation.

\section{PDAC tumor growth and metastasis depend on macrophage PI3K $\gamma$}

To investigate the functional role of macrophage PI3K $\gamma$ in pancreatic cancer, Panc02,

K8484, or p53 2.1.1 pancreatic tumor cells were orthotopically implanted in wild type or PI3K $\gamma$

deficient (p110 $-/-)$ mice, according to the depicted schema (Fig. 2A). Whereas WT

macrophages expressed PI3K $\gamma$, neither $\mathrm{p} 110 \gamma-/-$ macrophages nor PDAC cells expressed this

kinase (Fig. 2B and Supplementary Fig. 2A). While normal p110 $\gamma-/-$ pancreata were similar in

size as those from WT mice, pancreatic tumors from $\mathrm{p} 110 \gamma-/-$ mice were significantly smaller

than pancreatic tumors in WT mice, as p110 $\gamma$-/- tumors were reproducibly half the size of WT

tumors (Fig. 2C-E). Notably, the incidence of PDAC metastases, including kidney, diaphragm 
and liver metastases, was also significantly reduced in p110 $\gamma$-/- animals (Fig. 2F-G), indicating that macrophage PI3K $\gamma$ contributes to tumor spread.

We previously showed that PI3K $\gamma$ regulates the trafficking of bone marrow-derived $\mathrm{CD} 11 \mathrm{bGr} 1^{\text {lo }}$ monocytes and $\mathrm{CD} 11 \mathrm{bGr} 1^{\text {hi }}$ granulocytes into lung tumors by stimulating integrin $\alpha 4 \beta 1$ mediated adhesion to endothelium $(24-25,33)$. To determine if PI3K $\gamma$ regulates myeloid cell trafficking in pancreatic tumors, we performed flow cytometry to quantify immune cell populations in tissues from PDAC-bearing p110 $\gamma$-/- vs WT animals and in PI3K $\gamma$ inhibitor vs vehicle control-treated animals. Whereas PI3K $\gamma$ inhibition only slightly suppressed recruitment of CD11b+Gr1+ myeloid cells to tumors, it had little effect on the recruitment of CD11b+Gr1F480 ${ }^{\text {hi }}$ macrophages into PDACs (Fig. 2H-I; Supplementary Fig. S2B). Importantly, PDACs from p110 $\gamma-/-$ mice exhibited significantly more CD4+ and CD8+ T cell content than PDACs from WT mice (Figure 2J-K). In contrast, no differences were observed in the number of T cells in spleen, lung or liver of p110 $\gamma-/$ - and WT mice (Supplementary Fig. S2C-D). As p110 $\gamma$ is primarily expressed in myeloid cells, and p110 is the major isoform PI3K isoform in T cells (Figure 1F), these results suggest that PI3K $\gamma$ inhibition in myeloid cells promotes enhanced CD8+ T cell mobilization into PDACs, thereby enhancing anti-tumor immunity. 


\section{Genetic ablation of PI3k rimproves survival in mice with pancreatic adenocarcinomas}

To determine whether PI3K $\gamma$ inhibition improves the survival of mice with spontaneous

pancreatic tumors, we crossed PI3K $\gamma$-/- mice with $\mathrm{KC}$ and $\mathrm{KPC}$ mice (27-28). PI3K $\gamma$ deletion significantly delayed tumor progression and extended the survival of both $\mathrm{KC}$ and KPC mice

(Fig. 3A-B). The median survival time of control KC mice was 36 weeks, while median survival

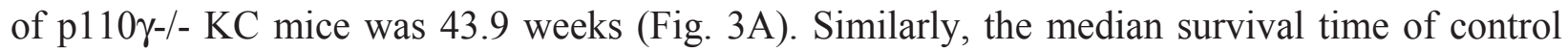
KPC animals was 26 weeks while the median survival time of p110 $\gamma-/-\mathrm{KPC}$ animals was 35.5 weeks (Fig. 3B). Remarkably, twenty percent of p110 $\gamma-/-$ KPC mice lived for more than one year, whereas none of the WT mice survived beyond 40 weeks. Analysis of pancreata from KPC animals by immunohistochemistry indicated that most KPC animals exhibited extensive claudin + , cytokeratin19+ invasive carcinoma (30) with little normal amylase+ pancreas remaining (Fig. 3C-E). WT animals almost uniformly exhibited metastases in organs that included liver, heart, lung and kidney (Fig. 3F). In contrast, KPC; p110 $\gamma$-/- animals exhibited extensive areas of normal amylase+ pancreas, little claudin+ and cytokeratin19+ invasive carcinoma (Fig. 3C-E), and little evidence of metastasis (Fig. 3F). Together, these results illustrate that PI3K $\gamma$ drives tumor development and spread in the pancreas and whereas PI3K $\gamma$ inhibition prevents these events and significantly extends survival. 


\section{Pharmacological inhibition of PI3K $\gamma$ suppresses PDAC growth and metastasis}

Since PI3K $\gamma$ promotes PDAC growth and spread, we speculated that pharmacological inhibitors of PI3K $\gamma$ could favorably impact the outcome of pancreas cancer. To test this, we evaluated the effect of the investigational PI3K $\gamma / \delta$ inhibitor TG100-115, which has an $\mathrm{IC}_{50}$ of $83 \mathrm{nM}$ for $\mathrm{p} 110 \gamma, 238 \mathrm{nM}$ for $\mathrm{p} 110 \delta$ and $>1000 \mathrm{nM}$ for $\mathrm{p} 110 \alpha$ and $\mathrm{p} 110 \beta$ (34), in mouse models of PDAC. mCherry-labeled LMP cells were orthotopically implanted into the pancreata of WT mice, and mice were then treated with the PI3K $\gamma$ inhibitor TG100-115 or chemically matched control compound, according to the schema depicted in Figure 4A. Intravital imaging revealed that control-treated mice exhibited large pancreatic tumors and multiple metastases, whereas TG100-115 treated mice had significantly smaller pancreatic tumors and few, if any metastases (Fig. 4A and Supplementary Fig. S3A-B). Quantification of tumor size by area of fluorescence and by tumor weight demonstrated that TG100-115 substantially suppressed PDAC growth to approximately half of that of control treated animals (Fig. 4B-C). TG100-115 also quantitatively suppressed metastasis to the diaphragm, liver, colon and other organs (Fig. 4D). Either early or late treatment with TG100-115 suppressed growth of p53 2.1.1 orthotopic tumors (Fig. 4E; Supplementary Fig. 4A-C), indicating PI3K $\gamma$ inhibition can suppress the growth of late stage 
tumors as well as early stage tumors. Histological analysis of tumors revealed that TG100-115 treated tumors exhibited enhanced tumor necrosis compared to control treated tumors (Fig. 4F); however, TG100-115 had no direct affect on viability of any PDAC cell line (Supplementary Fig. S4D-E). As PI3K $\gamma$ inhibition suppresses tumor growth without directly targeting tumor cells, these results suggested that PI $3 \mathrm{~K} \gamma$ inhibitors might effectively combine with standard of care chemotherapy, which targets tumor cells directly.

To evaluate the combinatorial effect of PI3K $\gamma$ inhibition and chemotherapy on PDAC growth and progression, we treated mice bearing orthotopic PDAC tumors with TG100115 and gemcitabine, according to the depicted schema (Fig. 4G). TG100-115 and gemcitabine similarly suppressed tumor growth as single agents, while the combination of the two treatments even further suppressed tumor growth (Fig. 4G) and significantly inhibited metastasis (Fig. 4H). Importantly, combined TG100-115 and gemcitabine treatment prolonged survival of mice with implanted PDACs from a median survival of 28 days for control animals to 38 days for the combination of Gemcitabine and TG100-115 (Fig 4I). Additionally, long-term TG100-115 treatment significantly increased survival of KPC mice with spontaneous pancreatic tumors and was accompanied by substantial reductions in metastasis (Fig. 4J-L). These results indicate that $\mathrm{PI} 3 \mathrm{~K} \gamma$ inhibitors provide long-term survival benefits in these models of pancreas cancer. 


\title{
PI3K $\gamma$ drives macrophage polarization and immune suppression in vitro and in vivo
}

\author{
Although myeloid cells can establish immunosuppressive microenvironments by

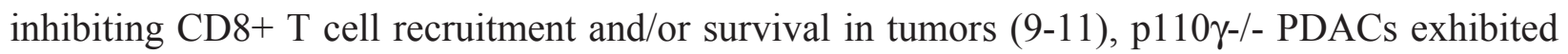 \\ increased CD8+ cell influx (Fig 2J-K). Therefore, we investigated whether PI3K $\gamma$ inhibition
} alters the immune response expression profile of pancreatic ductal adenocarcinomas. The expression of genes associated with immune suppression, chronic inflammation or tumor angiogenesis, including Arg1, Tgfb, Il1b, Il6 and Vegfa, were strongly expressed in myeloid cells isolated from WT PDAC tumors (Supplementary Fig. S5A). Genetic (p110 -/-) and pharmacological (TG100-115) inhibition of PI3K $\gamma$ both significantly inhibited expression of these genes in orthotopic PDAC tumors and in TAMs purified from PDAC tumors (Fig. 5A-B, Supplementary Fig. S5B-C). In contrast, the expression of immunostimulatory factors, including Il12 and Ifng, was significantly enhanced in tumors and TAMs from p110 $\gamma-/-$ and PI3K $\gamma$ inhibitor-treated animals (Fig. 5A-B, Supplementary Fig S5B-C). However, no significant difference in gene expression was observed in macrophages isolated from the uninvolved spleen or liver of WT and p110 $\gamma$-/- PDAC tumor bearing mice (Supplementary Fig S5D-E). To determine whether PI3K $\gamma$ directly controls these transcriptional changes in macrophages, we 
evaluated the effect of PI3K $\gamma$ inhibition in IL-4 polarized in vitro cultured macrophages, which model tumor derived macrophages, as they express immune-suppressive cytokines and factors, including Arg1, Il10 and Tgfb, and inhibit expression of immune stimulatory genes including Il12b and Ifng (35-39). Genetic or pharmacological inhibition of PI3K $\gamma$ not only inhibited immunosuppressive gene expression but also stimulated expression of Il12b, Ifng and Nos2 (Fig. 5C), indicating that PI3K $\gamma$ directly regulates a macrophage transcriptional switch from immune suppression toward immune stimulation.

To determine if selective blockade of PI3K $\gamma$ in macrophages was sufficient to disrupt tumor growth, we performed adoptive transfer studies by implanting tumor-derived macrophages in tumors grown in WT host mice. Tumor derived macrophages were isolated from WT and p110 $\gamma$-/- p53 2.1.1 tumors, mixed with freshly cultured p53 2.1.1 tumor cells and implanted in

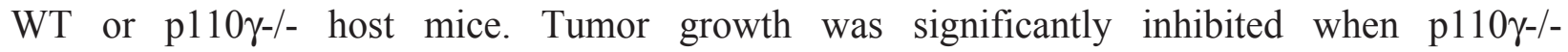
macrophages were implanted in either WT or p110 $/$-/- host mice (Fig. 5D). In contrast, tumor growth was enhanced when WT macrophages were implanted in p110 $\gamma$-/- host mice (Fig. 5D). Importantly, tumors implanted with p110 $\gamma-/-$, but not WT, macrophages exhibited a three-fold increase in CD8+ T cell content (Supplementary Fig. S5F). 
Our studies show that PI3K $\gamma$ inhibition alters macrophage transcriptional programming,

leading to increased CD8+ T cell recruitment and reduced PDAC growth (Fig. 2; Supplementary

Fig. S5F). We speculated therefore, that PI3K $\gamma$ inhibition indirectly activates $\mathrm{T}$ cell mediated anti-tumor immune responses in vivo. Accordingly, we found that T cells isolated from p110 $\gamma-/-$ PDAC tumors exhibited enhanced expression of the $\mathrm{T}_{\mathrm{H}} 1$ cytokine Ifng and downregulated expression of the immune suppressive cytokines Tgfb and Il10 (Fig. 5E). To exclude the possibility that PI3K $\gamma$ within T cells directly controls $\mathrm{T}$ cell activation, we examined the effect of p110 $\gamma$ inhibition on $\mathrm{T}$ cell proliferation ex vivo. $\mathrm{T}$ cells were isolated from naive (Fig. 5F) or PDAC-bearing (Fig. 5G) WT and p110 $\gamma-/-$ mice, and proliferation was stimulated by incubation with anti-CD3, anti-CD3+CD28 or IL2 + anti-CD3+CD28 antibodies. No differences were observed in the proliferative capacity of WT and p110 $\gamma-/-\mathrm{T}$ cells, whether from naive or tumorbearing mice. To determine if the anti-tumor effect of p110 $\gamma$ inhibition required the presence of

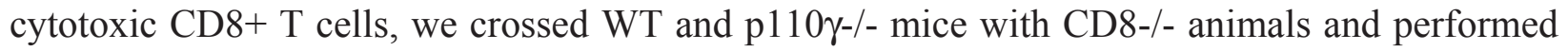
PDAC tumor studies. As only 3\% of live cells in WT PDACs were CD8+ T cells (Fig. 2J), it was not surprising that CD8 deletion had little effect on WT PDAC tumor size (Fig 5H). Importantly, CD8 deletion ablated the tumor suppressive effect of $\mathrm{p} 110 \gamma$ inhibition (Fig. $5 \mathrm{H}$ ). Taken together, these results indicate that macrophage PI3K $\gamma$ suppresses $\mathrm{T}$ cell mediated immune surveillance by promoting expression of immunosuppressive cytokines by tumor associated macrophages. 
Furthermore, our studies indicate that PI3K $\gamma$ inhibition indirectly stimulates $\mathrm{T}$ cell mediated antitumor immune responses, leading to growth suppression in PDAC.

Recent progress in anti-cancer therapeutics has led to the development and clinical approval of $\mathrm{T}$ cell checkpoint inhibitors for therapy of some solid tumors, including melanoma and lung carcinoma (18-21). To explore the possibility that PI3K $\gamma$ inhibitors might synergize with $\mathrm{T}$ cell checkpoint inhibitors for the treatment of PDACs, we first evaluated expression of the programmed cell death protein-1 (PD-1) and its ligand, programmed cell death ligand-1 (PDL1) in WT and p110y-/- PDAC tumors. Although PD-1 was expressed on CD8+ T cells from PDAC tumors, we observed no significant differences in expression levels in T cells from WT

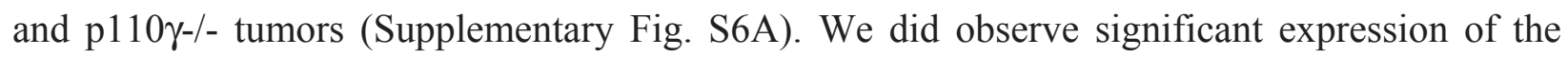
checkpoint ligand PD-L1 on macrophages but not tumor cells from PDACs but no difference in expression between cells from WT and p110 $\gamma$-/- tumors (Supplementary Fig. S6B). We also observed no change in the presence of FoxP3+CD25+CD4+ T regulatory cells in WT and p110 $\gamma-$ /- tumors (Supplementary Fig. S6C). To explore the relative effects of PI3K $\gamma$ inhibition and $\mathrm{T}$ cell checkpoint inhibition as therapeutic strategies, we treated WT and p110 $\gamma^{-/-}$animals bearing PDAC tumors with anti-PD-1 or isotype control antibodies. Both anti-PD-1 and p110 $\gamma$ inhibition substantially suppressed PDAC tumor growth (Supplementary Fig. S6D-E). However, the 
combination of anti-PD-1 with p110 $\gamma$ inhibition provided no added benefit over single agent

therapy (Supplementary Fig. S6D-E). We also treated WT animals bearing PDAC tumors with

TG100-115, anti-PD-1, isotype control antibodies or combination therapy; similarly no benefit was observed with the combined treatment. These data support the conclusion that PI3K $\gamma$ inhibition promotes an anti-tumor $\mathrm{T}$ cell response that, like anti-PD-1, relieves $\mathrm{T}$ cell exhaustion. Future studies optimizing checkpoint inhibitor immunotherapy and tumor cell targeted therapy in combination with $\mathrm{PI} 3 \mathrm{~K} \gamma$ inhibition may provide further insights into the nature of immune suppression in PDAC cancer.

\section{PI3K ycontrols macrophage PDGF expression to promote PDAC cell invasion}

PI3K $\gamma$ inhibition mediated a striking suppression of metastasis in orthotopic and spontaneous PDAC models (Fig. 2-4). To determine whether macrophage PI3K $\gamma$ promotes PDAC invasion, we analyzed the effect of conditioned medium (CM) from IL-4 stimulated WT or p110 $\gamma-/-$ macrophages on migration of LMP and p53 2.1.1. PDAC cells. CM from WT macrophages stimulated robust chemotaxis, while CM from $\mathrm{p} 110 \gamma-/-$ macrophages was less effective in stimulating cell migration (Fig. 6A; Supplementary Fig. S7A). Macrophage CM had no effect on cell proliferation (Supplementary Fig. S7B). To identify factors that could account 
for these differences in migration, we performed RNA sequencing on p110 $\gamma-/-$ and WT IL-4 stimulated macrophages and found that the mRNA expression of many chemotactic factors, including Ccl2, Scf, Hbegf, Pdgfa, and Pdgfb, was strongly suppressed in p110 $\gamma$-/- macrophages

(Fig. 6B). To determine if these factors could stimulate PDAC invasion, we tested the effect of the PDGFR inhibitor Imatinib (40-41), the PDGF inhibitor Fovista (42), the EGFR inhibitors

Erlotinib (43), Lapatinib (44) and anti-CCL2 on CM stimulated chemotaxis. Only Imatinib and Fovista suppressed PDAC migration toward macrophage CM, indicating that PDGF and PDGFR are required for macrophage stimulated PDAC chemotaxis (Fig. 6C-D and Supplementary Fig. S7C-D). To determine which cytokines were sufficient to induce migration, we supplemented p110 - -/- CM with PDGF-AA, PDGF-BB, SCF or CCL2 and found that only PDGF-BB was sufficient to restore chemotactic migration in murine and human PDAC cells (Fig. 6E; Supplementary Fig. S7E-F). Notably, we found that PDGF-BB protein expression was reduced in $\mathrm{p} 110 \gamma-/-\mathrm{CM}$ and in PDAC tumors from $\mathrm{p} 110 \gamma-/-$ mice (Fig. 6F-G). As p110 $\gamma-/-\mathrm{CD} 11 \mathrm{~b}+\mathrm{Gr} 1-$ tumor derived macrophages expressed reduced $P D G F \beta$ mRNA in vivo (Fig. $6 \mathrm{H}$ ), our studies show that macrophage derived PDGF-BB may promote PDAC metastasis in vivo and that PI3K $\gamma$ inhibitors may block metastasis by suppressing macrophage expression of PDGF-BB.

\section{PI3K rmediated macrophage PDGF-BB expression promotes desmoplasia in PDAC}


Pancreatic tumors are associated with extensive desmoplasia; this severe desmoplastic response impedes the effectiveness of chemotherapy and disrupts the normal functions of the pancreas (45). Pancreata from $\mathrm{p} 110 \gamma-/-\mathrm{KC}$ and KPC animals exhibited substantially less desmoplasia than their WT counterparts, as revealed by Masson's Trichrome staining of pancreatic tumor sections (Fig. 7A). Similarly, pancreata from KPC animals treated with the PI3K $\gamma$ inhibitor TG100-115 exhibited less desmoplasia by Trichrome staining (Fig. 7B). Similar differences in desmoplastic response were seen in picrosirius red stained pancreata from KPC orthotopic tumors grown in WT and p110 -/- animals (Fig. 7C-D). Additionally, less collagen protein and gene expression was observed in orthotopic LMP tumors that were treated with the PI3K $\gamma$ inhibitor TG100-115 (Fig. 7E-G). To determine whether macrophage PI3K $\gamma$ regulates fibroblast-mediated collagen expression, we incubated primary murine fibroblasts with CM from basal and IL-4 stimulated WT and p110 $\gamma-/-$ macrophages. Less collagen mRNA was expressed in

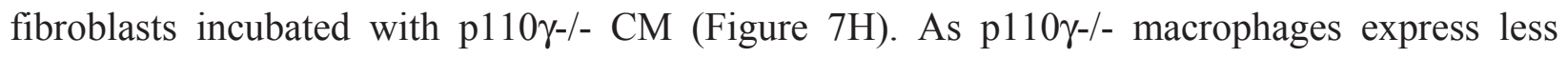
TGF $\beta 1$ and PDGF-BB than WT macrophages, we tested the effect of inhibitors of these factors in collagen expression assays. Only anti-PDGF-BB and Imatinib suppressed collagen expression in fibroblasts, indicating that macrophage PI3K $\gamma$ likely regulates desmosplasia associated with PDAC by controlling expression of secreted PDGF-BB (Fig. 7I). 
In summary, the studies presented here demonstrate the critical roles that PI3K $\gamma$ plays in regulating PDAC tumor growth and progression. Our studies demonstrate PI $3 \mathrm{~K} \gamma$ promotes transcription of genes associated with the M2 immunosuppressive macrophage phenotype in PDACs, including immune suppressive factors such as Arg1, Tgfb, and Il10 and chemokines and wound healing factors that include PDGF-BB. By inhibiting PI3K $\gamma$, the expression of these genes is constrained, thereby activating CD8+ T cell dependent tumor suppression and increased survival. 


\section{Discussion}

Inflammation plays a critical role in pancreatic carcinoma progression and relapse from

therapy (1-4). B cells, T cells and myeloid cells can all be found in the pancreatic tumor microenvironment, yet these cells do not mount an appropriate anti-tumor adaptive immune response. It is now accepted that tumor associated macrophages and myeloid derived suppressor cells release immune suppressive factors that inhibit $\mathrm{T}$ cell mediated anti-tumor responses (3, 38), and therapeutic approaches that are aimed at preventing myeloid derived suppressive responses show some therapeutic efficacy in animal models of cancer (12-17). T cell checkpoint inhibitors that target $\mathrm{T}$ cell functions in cancer also hold some promise as novel therapeutics for pancreatic cancer (18-21). However, strategies that inhibit myeloid cell-mediated immune suppression may well boost the effect of checkpoint inhibitors, vaccines and other therapeutic strategies in the highly immunosuppressive microenvironment of pancreatic tumors.

In the studies presented here, we have identified PI3K $\gamma$ as a critical regulator of the pathways that control immune suppression, metastasis and desmoplasia in pancreatic cancer. We showed that PI3K $\gamma$ is expressed in human and murine pancreatic tumor macrophages and that selective deletion of this PI3K isoform suppresses orthotopic and spontaneous PDAC growth and 
metastasis. In addition, PI3K $\gamma$ inhibition significantly enhanced survival of mice bearing spontaneous PDACs by suppressing tumor growth and metastasis. Our studies demonstrate that $\mathrm{PI} 3 \mathrm{~K} \gamma$ plays a key role in activating the immune suppressive transcriptional signature of tumor derived macrophages, in that PI3K $\gamma$ inhibition suppressed expression of Arg1, Tgfb, and Il10 and stimulated expression of Il12 and Ifng in vivo. These changes in myeloid gene expression signatures were associated with increased CD8+ T cell recruitment to PDAC tumors, increased T cell expression of IFN $\gamma$ and decreased expression of TGF $\beta$ and IL10, and CD8+ T cell dependent tumor suppression. Our results are in agreement with recent studies that showed that PI3K $\delta$ but not $\mathrm{PI} 3 \mathrm{~K} \gamma$ is required for $\mathrm{T}$ cell activation but contrast with studies that showed that $\mathrm{p} 110 \gamma$ is required for $\mathrm{T}$ cell recruitment by inflammatory chemokines (46-49). It is possible that $\mathrm{T}$ cell recruitment to tumors in vivo depends on chemokines that only activate p110 $\delta$ rather than p110 $\gamma$. Finally, we show that $\mathrm{PI} 3 \mathrm{~K} \gamma$ also regulates macrophage expression of PDGF-BB, which stimulates tumor cell chemotaxis and fibroblast production of collagen in vitro and in vivo. Recent studies revealed that p53 mutations induce constitutive PDGFR expression and signaling that drives tumor invasion and metastasis (50). Our studies suggest that macrophage derived PDGF-BB may cooperate with this mutant pathway to promote metastasis but also show that targeting PI3K $\gamma$ can control this pathway by which PDACs spread. Our studies thus demonstrate 
that inhibitors of PI3K $\gamma$ offer promise as new therapeutic approaches to control tumor growth and progression, metastasis and desmoplasia in this devastating malignancy.

In related studies, we demonstrated that human and murine PDACs exhibit increased

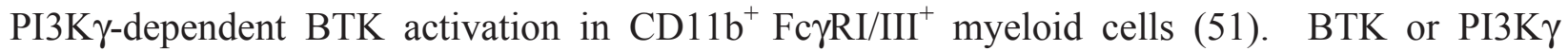
inhibition as monotherapy in early stage PDAC, or in combination with Gem in late-stage PDAC slowed progression of orthotopic tumors in a manner dependent on T cells (51). However, we

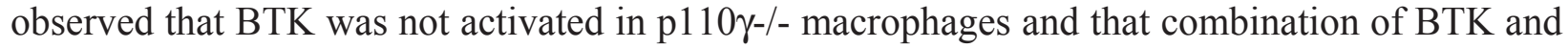
PI3K $\gamma$ inhibitors had no additive effects in regulating macrophage gene expression or PDAC progression. These studies indicated that the two kinases regulate overlapping signal transduction pathways in macrophages. An increase in effector and memory $\mathrm{CD}^{+} \mathrm{T}$ cell phenotypes was also observed in these studies, which is consistent with other reports about $\mathrm{CD}^{+} \mathrm{T}$ cell responses to various immunotherapies (17).

We demonstrated that genetic and pharmacological PI3K $\gamma$ inhibition was as effective as treatment with the checkpoint inhibitor anti-PD-1 in mouse models of PDAC. Although we were unable to observe additive effects of targeting PI3K $\gamma$ and PD-1 on tumor growth at this time, we did observe improved survival by combining PI3K $\gamma$ inhibitors and chemotherapeutic agents. Our 
studies show that targeted inhibition of PI3K $\gamma$ can combine with other therapeutic approaches targeting distinct components of the tumor microenvironment to effect long-term durable anti-

PDAC tumor immune responses. Research. 


\section{Methods}

Institutional approvals: All studies with human tissues were approved by the Institutional Review Board for human subjects research of the University of California, San Diego (UCSD). Informed consent was obtained from all patients prior to surgery. The use of samples occurred under " "exempt category 4" for research on de-identified biological specimens. All animal experiments were performed with approval from the Institutional Animal Care and Use Committees of the University of California, San Diego and the University of Torino, Italy.

Reagents: TG100-115 was from Targegen, Inc. (La Jolla, CA). Fovista was from Ophthotech (New York, NY).

Cell lines: The p53 2.1.1 pancreatic adenocarcinoma cell line was derived from primary PDAC tumors $(\mathrm{Fvb} / \mathrm{N})$ of male transgenic $\mathrm{KC}$ mice harboring null mutations in p53. The LMP pancreatic adenocarcinoma cell line was derived from a liver metastasis of a primary PDAC tumor from transgenic KPC mice in a C57B16;129Sv background. The K8484 pancreatic adenocarcinoma cell line was derived from a primary PDAC tumor from transgenic KPC mice in a C57B16 background. The Panc02 cell line from C57B16 mice has been previously described 
(33). All cell lines were tested for mycoplasma contamination and grown in DMEM/10\% FBS/1.0\% Pen-Strep on plastic plates (LMP, Panc02, K8484) or plastic plates coated with $50 \mu \mathrm{g} / \mathrm{ml}$ rat tail collagen I (p53 2.1.1)(BD Biosciences). Cells used in these studies were authenticated by morphological profiling and RNA sequencing and RT-PCR in 2012, whole exome analysis in 2015 (p53 2.1.1), and PI3K $\gamma$ inhibitor sensitivity analyses (2013-2015). Panc02 cells and LMP were acquired in 2009; K8484 cells and p53 2.1.1 cells were acquired in 2014.

Animals: Generation and characterization of $\mathrm{p} 110 \gamma-/-, \mathrm{KC}$ and KPC mice have been described previously $(23-24,29)$. KPC animals in the C57Bl6 background were crossed with Pik3cg-/animals in the C57Bl6 background to generate Pik3cg-/-KC; and Pik3cg-/-; KPC mice in the Animal Facility at the Molecular Biotechnology Center, University of Turin.

Tumor studies: Ten thousand p53 2.1.1 PDAC cells were orthotopically implanted into the pancreata of $\mathrm{p} 110 \gamma+/+(\mathrm{WT})$ or $\mathrm{p} 110 \gamma-/-8-10$ week old FVB/n mice. Five hundred thousand LMP PDAC cells were orthotopically implanted into the pancreata of 8-10 week old C57B16;129 mice and five hundred thousand K8484 or Panc02 PDAC cells were orthotopically implanted into the pancreata of 8-10 week old C57B16 mice $(n=10)$. In some studies, WT and p110 $\gamma-/-$ 
animals with tumors were treated by i.p injection with and without gemcitabine $(150 \mathrm{mg} / \mathrm{kg}) \mathrm{on}$ $\mathrm{d} 7$ and $\mathrm{d} 14(\mathrm{n}=10)$. In other studies, mice were treated by i.p injection b.i.d. with $2.5 \mathrm{mg} / \mathrm{kg}$ of PI3K $\gamma$ inhibitor (TG100-115) or with a chemically similar inert control twice daily from day 7$21(\mathrm{n}=10)$. In some studies, WT and $\mathrm{p} 110 \gamma-/-$ mice were treated with $100 \mu \mathrm{g}$ anti-PD-1 or isotype control clone LTF-2, (BioXCell) administered by i.p. injection on day 7, 10 and 13 of tumor growth. Mice were sacrificed on d21. For all tumor experiments, tumor volumes and weights were recorded at sacrifice. In other studies, the growth, metastasis and survival of spontaneous

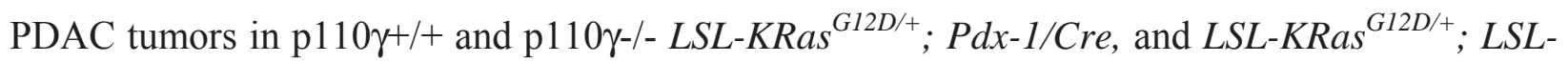
$\operatorname{Trp} 53^{R 172 H /+} \mathrm{Pd} x-1$ Cre animals was evaluated over $100+$ weeks.

RNA sequencing: Freshly isolated mouse bone marrow cells from 9 WT and 9 p110 $\gamma-/-$ mice were pooled into 3 replicates sets of WT or p110 $\gamma$-/- cells that were differentiated into macrophages for six days in RPMI $+20 \% \mathrm{FBS}+1 \% \mathrm{Pen} / \mathrm{Strep}+50 \mathrm{ng} / \mathrm{ml} \mathrm{M}-\mathrm{CSF}$. Each replicate set of macrophages was then incubated for $48 \mathrm{~h}$ with mCSF or mCSF + IL-4. Macrophages were removed from dishes, and RNA was harvested using Qiagen Allprep kit. One microgram of total RNA per sample was used for the construction of sequencing libraries. RNA sequencing was performed by the University of California, San Diego Institute for Genomic Medicine Genomics Center as follows: RNA libraries were prepared for sequencing using standard Illumina 
protocols. mRNA profiles of MCSF and IL4 stimulated macrophage derived from wild-type (WT) and PI3Kinase gamma null (p110 $-/-)$ mice were generated by single read deep sequencing, in triplicate, using Illumina HiSeq2000. Sequence analysis was performed as previously described and results are available to view online at the NCBI Gene Express Omnibus website (file number GSE58318).

Statistics: For studies evaluating the effect of drugs on tumor growth, a sample size of at least 10 mice/group provided $80 \%$ power to detect mean difference of 2.25 standard deviation (SD) between two groups (based on a two-sample t-test with 2-sided 5\% significance level). Prior to statistical analyses, data were examined for quality and possible outliers. Data were normalized to the standard where applicable. Significance testing was performed by one-way Anova with Tukey's posthoc testing for multiple pairwise testing or by parametric or nonparametric Student's $t$ test as appropriate. Fisher's exact test was used to query significant differences in rates of metastasis between groups.

Supplementary methods: Additional detailed methods are available online in Supplementary Materials. 


\section{Acknowledgments}

The authors thank Ophthotech for the gift of Fovista and Sanofi-Aventis/Targegen for the gift of

TG100-115. The authors also thank Xiaodan Song, Joan Manglicmot and Roberta Curto for technical assistance.

Grant support: The authors acknowledge support from T32HL098062 to MMK and R01CA167426-03S1 to AVN; from Ministero della Salute Ricerca Sanitaria Finalizzata RF2013-02354892, Associazione Italiana Ricerca sul Cancro (5 x mille no. 12182) and (IG no. 15257); University of Turin-Progetti Ateneo 2014-Compagnia di San Paolo (PANTHER to PC) (PC-METAIMMUNOTHER) to FN and support from the NCI/NIH (R01CA167426, R01CA126820 and R01CA083133), Lustgarten Foundation and AACR/Landon Foundation to JAV.

Author contributions: $\mathrm{MMK}, \mathrm{CH}$ and PS performed $\mathrm{IHC}$ analysis. MMK, $\mathrm{CH}, \mathrm{PF}, \mathrm{PC}$ and MCS performed orthotopic PDAC models. EM, MMK, MCS and PC performed PDAC GEMM studies. MMK and $\mathrm{CH}$ performed Western blotting. MMK, AVN, NR, CH, SG, PF and MCS performed RT-PCR. Cell migration studies were performed by AVN and MMK. Collagen expression analysis was performed by AVN and MMK. MV provided human pancreas tissues 
and pathological analysis. RS performed RNA seq data analysis. MB, AL and JAV directed studies at UCSD. FN and EH directed studies at University of Torino. JAV, MB, AML, FN and EH conceived the studies. JAV wrote the manuscript. 


\section{References}

1. Liou GY, Storz P. Inflammatory macrophages in pancreatic acinar cell metaplasia and initiation of pancreatic cancer. Oncoscience 2015; 2:247-51.

2. Clark CE, Hingorani SR, Mick R, Combs C, Tuveson DA, Vonderheide RH. Dynamics of the immune reaction to pancreatic cancer from inception to invasion. Cancer Res 2007; 67:9518-27.

3. Karakhanova S, Link J, Heinrich M, Shevchenko I, Yang Y, Hassenpflug M, et al. Characterization of myeloid leukocytes and soluble mediators in pancreatic cancer: importance of myeloid-derived suppressor cells. Oncoimmunology 2015; 4:e998519.

4. Beatty GL, Winograd R, Evans RA, Long KB, Luque SL, Lee JW, et al. Exclusion of T Cells From Pancreatic Carcinomas in Mice Is Regulated by Ly6C(low) F4/80(+) Extratumoral Macrophages. Gastroenterology 2015; 149:201-10.

5. SEER.cancer.gov [Internet]. Rockville: National Cancer Institute; c2015. Available from: http://seer.cancer.gov/statfacts/html/pancreas.html. 
6. Siegel R, Naishadham D, Jemal A. Cancer statistics 2012. CA Cancer J Clin 2012; 62; 10-29.

7. Conroy T, Desseigne F, Ychou M, Bouche O, Guimbaud R, Becouarn Y, et al. FOLFIRINOX versus gemcitabine for metastatic pancreatic cancer. $N$ Engl J Med 2011; 364:1817-25.

8. Von Hoff DD, Ramanathan RK, Borad MJ, Laheru DA, Smith LS, Wood TE, et al. Gemcitabine plus nab-paclitaxel is an active regimen in patients with advanced pancreatic cancer: a phase I/II trial. J Clin Oncol 2011; 29: 4548-54.

9. Ruffell B and Coussens LM. Macrophages and Therapeutic Resistance in Cancer. Cancer Cell 2015; 27: 462-72.

10. Schmid MC, Varner JA. Myeloid cells in tumor inflammation. Vasc Cell 2012; 4:14.

11. Coussens LM, Zitvogel L, Palucka AK, Neutralizing tumor-promoting chronic inflammation: a magic bullet? Science 2013; 339: 286-91. 
12. Stortz P. The crosstalk between acinar cells with Kras mutations and M1-polarized macrophages leads to initiation of pancreatic precancerous lesions. Oncoimmunology 2015; 4: e1008794.

13.Stromnes IM, Brockenbrough JS, Izeradjene K, Carlson MA, Cuevas C. Targeted depletion of an MDSC subset unmasks pancreatic ductal adenocarcinoma to adaptive immunity. Gut 2014: 63:769-81.

14. Beatty GL, Chiorean EG, Fishman MP, Saboury B, Teitelbaum UR, Sun W, et al., CD40 agonists alter tumor stroma and show efficacy against pancreatic carcinoma in mice and humans. Science 2011; 331:1612-16.

15. Zhu Y, Knolhoff BL, Meyer MA, Nywening TM, West BL, Luo J. CSF1/CSF1R blockade reprograms tumor-infiltrating macrophages and improves response to T-cell checkpoint immunotherapy in pancreatic cancer models. Cancer Res 2014; 74:5057-69. 
16. Chu NJ, Armstrong TD, Jaffee EM. Nonviral oncogenic antigens and the inflammatory signals driving early cancer development as targets for cancer immunoprevention. Clin Cancer

Res 2015. 21:1549-57.

17. Cappello P, Rolla S, Chiarle R, Principe M, Cavallo F, Perconti G, et al. Vaccination with ENO1 DNA prolongs survival of genetically engineered mice with pancreatic cancer. Gastroenterology 2013; 144:1098-106.

18. Keenan BP, Saenger Y, Kafrouni MI, Leubner A, Lauer P, Maitra A et al. A Listeria vaccine and depletion of T-regulatory cells activate immunity against early stage pancreatic intraepithelial neoplasms and prolong survival of mice. Gastroenterology 2014; 146:1784-94 e6.

19. Twyman-Saint Victor C, Rech AJ, Maity A, Rengan R, Pauken KE, Stelekati E, et al., Radiation and dual checkpoint blockade activate non-redundant immune mechanisms in cancer.

Nature 2015. 520:373-7.

20. Sharma P and Allison JP. The future of immune checkpoint therapy. Science 2015; 348:56-

61. 
21. Topalian SL, Drake CG, Pardoll DM. Immune Checkpoint Blockade: A Common Denominator Approach to Cancer Therapy. Cancer Cell 2015; 27:450-461.

22. Martini M, De Santis MC, Braccini L, Gulluni F and Hirsch E. PI3K/AKT signaling pathway and cancer: an updated review. Ann Med 2014; 46: 372-383.

23. Vanhaesebroeck B, Stephens L and Hawkins P. PI3K signalling: the path to discovery and understanding. Nat Rev Mol Cell Biol 2012; 13:195-203.

24. Schmid MC, Avraamides CJ, Dippold HC, Franco I, Foubert P, Ellies LG, et al., Receptor tyrosine kinases and TLR/IL1Rs unexpectedly activate myeloid cell PI3Kgamma, a single convergent point promoting tumor inflammation and progression. Cancer Cell, 2011. 19:715-7.

25. Schmid M C et al. PI3-kinase gamma promotes Rapla-mediated activation of myeloid cell integrin alpha4beta1, leading to tumor inflammation and growth. PLoS One 2013; 8: e60226. 
26. Zeng L, Guo Y, Liang J, Chen S, Peng P, Zhang Q, et al. Perineural Invasion and TAMs in

Pancreatic Ductal Adenocarcinomas: Review of the Original Pathology Reports Using Immunohistochemical Enhancement and Relationships with Clinicopathological Features. $J$ Cancer 2014; 5:754-60.

27. Tuveson DA, Shaw AT, Willis NA, Silver DP, Jackson EL, Chang S, et al. Endogenous oncogenic K-ras(G12D) stimulates proliferation and widespread neoplastic and developmental defects. Cancer Cell 2004; 5:375-87.

28. Hingorani SR, Wang L, Multani AS, Combs C, Deramaudt TB, Hruban RH, et al. Trp53R172H and KrasG12D cooperate to promote chromosomal instability and widely metastatic pancreatic ductal adenocarcinoma in mice. Cancer Cell 2005; 7: 469-83.

29. Bardeesy N, Aguirre AJ, Chu GC, Cheng KH, Lopez LV, Hezel AF, et al. Both p16(Ink4a) and the p19(Arf)-p53 pathway constrain progression of pancreatic adenocarcinoma in the mouse.

Proc Natl Acad Sci U S A 2006; 103: 5947-52. 
30. Collins MA, Benar F, Zhang Y, Brisset J-C, Galban S, Galban, CJ et al. Oncogenic Kras is required for both the initiation and maintenance of pancreatic cancer in mice. J Clin Invest 2012;

122: $639-53$.

31. Collins MA, Brisset J-C, Zhang Y, Bednar F, Pierre J, et al. Metastatic pancreatic cancer is dependent on oncogenic Kras in mice. PLoS One 2012; 7:e49707.

32. Tseng WW, Winer D, Kenkel JA, Choi O, Shain AH, Pollack JR, et al. Development of an orthotopic model of invasive pancreatic cancer in an immunocompetent murine host. Clin Cancer Res 2010; 16:3684-95.

33. Schmid MC, Avraamides CJ, Foubert P, Shaked Y, Kang SW, Kerbel RS, et al. Combined blockade of integrin-alpha4beta1 plus cytokines SDF-1alpha or IL-1beta potently inhibits tumor inflammation and growth. Cancer Res 2011; 71:6965-75.

34. Palanki MS, Dneprovskaia E, Doukas J, Fine RM, Hood J, Kang X, et al., Discovery of 3,3'(2,4-diaminopteridine-6,7-diyl)diphenol as an isozyme-selective inhibitor of PI3K for the 
treatment of ischemia reperfusion injury associated with myocardial infarction. $J$ Med Chem 2007; 50:4279-4294.

35. Biswas SK, Gangi L, Paul S, Schioppa T, Saccani A, Sironi M, et al. A distinct and unique transcriptional program expressed by tumor-associated macrophages (defective NF-kappaB and enhanced IRF-3/STAT1 activation). Blood 2006; 107:2112-22.

36. Flavell RA, Sanjabi S, Wrzesinski SH, and Licona-Limon P. The polarization of immune cells in the tumour environment by TGFbeta. Nat Rev Immunol 2010; 10:554-67.

37. Barron L, Smith AM, El Kasmi KC, Qualls JE, Huang X, Cheever A, et al. Role of arginase 1 from myeloid cells in th2-dominated lung inflammation. PLoS One 2013; 8: e61961.

38. Rodriguez PC, Quiceno DG, Zabaleta J, Ortiz B, Zea AH, Piazuelo MB, et al. Arginase I production in the tumor microenvironment by mature myeloid cells inhibits T-cell receptor expression and antigen-specific T-cell responses. Cancer Res 2004; 64: 5839-49. 
39. El Kasmi KC, Qualls JE, Pesce JT, Smith AM, Thompson RW, Henao-Tamayo M, et al.

Toll-like receptor-induced arginase 1 in macrophages thwarts effective immunity against intracellular pathogens. Nat Immunol 2008; 9:1399-1406.

40. George D. Platelet-derived growth factor receptors: a therapeutic target in solid tumors. Semin Oncol 2001; 28:27-33.

41. Joensuu H, Dimitrijevic S. Tyrosine kinase inhibitor imatinib (STI571) as an anticancer agent for solid tumours. Ann Med 2001; 33:451-5.

42. Tolentino MJ, Dennrick A, John E, Tolentino MS. Drugs in Phase II clinical trials for the treatment of age-related macular degeneration. Expert Opin Investig Drugs 2015; 24:183-99.

43. Kim TE, Murren JR. Erlotinib OSI/Roche/Genentech. Curr Opin Investig Drugs 2002;

3:1385-95. 
44. Xia W, Mullin RJ, Keith BR, Liu LH, Ma H, Rusnak DW, et al. Anti-tumor activity of GW572016: a dual tyrosine kinase inhibitor blocks EGF activation of EGFR/erbB2 and downstream Erk1/2 and AKT pathways. Oncogene 2002; 21:6255-63.

45. Shi C, Washington KM, Chaturvedi R, Drosos Y, Revetta FL, Weaver CJ, et al. Fibrogenesis in pancreatic cancer is a dynamic process regulated by macrophage-stellate cell interaction. Lab Invest 2014; 94:409-21.

46. Alcazar I, Marques M, Kumar A, Hirsch E, Wymann M, Carrera AC et al. Phosphoinositide 3-kinase gamma participates in T cell receptor-induced T cell activation. J Exp Med 2007; 204: 2977-2987.

47. Martin AL, Schwartz M D, Jameson SC, Shimizu Y. Selective regulation of CD8 effector T cell migration by the p110 gamma isoform of phosphatidylinositol 3-kinase. J Immunol 2008;

180: 2081-2088. 
48. Ali K, Soond D R, Pineiro R, Hagemann T, Pearce W, Lim EL et al. Inactivation of PI(3)K

p110delta breaks regulatory T-cell-mediated immune tolerance to cancer. Nature 2014; 510 :

407-411.

49. Soond DR, Bjørgo E, Moltu K, Dale VQ, Patton DT, Torgersen KM et al. PI3K p110delta regulates T-cell cytokine production during primary and secondary immune responses in mice and humans. Blood 2010; 115: 2203-2213.

50. Weismueller S, Manchado E, Saborowski M, Morris J, Wagenblast E, Davis CA, et al. Mutant p53 drives pancreatic cancer metastasis through cell-autonomous PDGF Receptor $\beta$ signaling. Cell 2014; 157: 382-94.

51. Gunderson AJ Kaneda MM, et al. Bruton Tyrosine Kinase-Dependent Immune Cell Crosstalk Drives Pancreas Cancer. Cancer Discov, 2016; 6:270-285. 


\section{Figure Legends}

\section{Figure 1: PI3K $\gamma$ is a marker of pancreatic ductal adenocarcinoma associated macrophages}

A. Immunofluorescent staining of F4/80+ macrophages (red) counterstained with DAPI (blue) in

pancreata from normal mice, LSL-KRas ${ }^{G 12 D}$; Pdx-1Cre (KC) mice, LSL-KRas ${ }^{G 12 D /+}$; LSL$\operatorname{Trp}_{53^{R 172 H /+}}$; Pdx-1Cre (KPC) mice and mice that were implanted orthotopically with LSL-

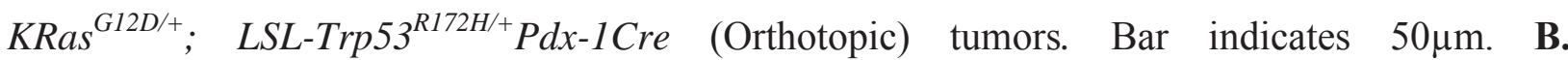
Quantification of the increase in $\mathrm{F} 4 / 80+$ macrophages over time in pancreata that were orthotopically implanted with LMP tumor cells $(n=10),{ }^{*} p<0.01$. C. Representative histopathologic characterization of human invasive pancreatic ductal adenocarcinoma tissues $(n=7)$ and normal pancreas $(n=8)$ showing immune reactivity for the macrophage marker CD68, with hematoxylin counterstaining. Scale bar indicates $100 \mu \mathrm{m}$. D. Quantification of CD68+ macrophages/100X microscopic field in tissue sections from normal human pancreata $(\mathrm{n}=7)$ and from invasive pancreatic ductal adenocarcinomas $(n=8)$. Statistical significance was determined via Wilcoxon rank-sum test. E. Immunostaining of human invasive pancreatic ductal adenocarcinomas for expression of PI3K $\gamma$ (green) and CD68+ macrophages (red). Tissues were counterstained with DAPI (blue) to detect nuclei. Arrows indicate examples of overlap (yellow)

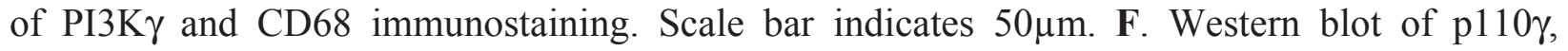




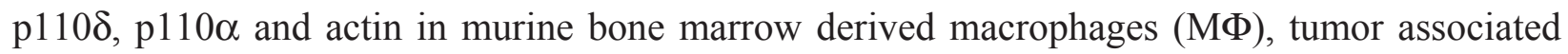
macrophages (TAM), CD19+ B cells, CD90+ T cells and LMP and p53 2.1.1 murine PDAC cells.

\section{Figure 2: PDAC tumor growth and metastasis depend on macrophage PI3K $\gamma$.}

A. Panc02, K8484 or p53 2.1.1 PDAC tumor cells were orthotopically implanted into syngeneic

WT or $\mathrm{p} 110 \gamma-/-$ mice according to the depicted schema. B. Western blot comparing p110 $\gamma$ and actin expression in WT and p110 $\gamma$-/- macrophages and in vitro cultured p53 2.1.1 PDAC cells.

C-E. Weights of normal pancreata as well as pancreata containing tumors from WT and p110 $\gamma-/-$ mice 28 days after orthotopic implantation with (C) Panc02, (D) K8484 or (E) p53 2.1.1 PDAC cells. Significance testing was performed by nonparametric $t$ test. F. Incidence of kidney, diaphragm and liver metastases in WT and p110 $\gamma$-/- animals implanted with Panc02 cells. Significance testing was performed by Fisher's exact test. G. Images of kidney, diaphragm and liver metastases from F. Arrowheads indicate metastatic foci. Scale bar indicates $50 \mu \mathrm{m}$. H. Quantification of CD11b+Gr1 - macrophages and CD11b+Gr1+ myeloid cells by flow cytometry in representative tumors from WT $(n=3)$ and $p 110 \gamma-/-(n=5)$ mice. Significance testing was performed by unpaired $t$ test. I. Representative Facs plots from WT and p110 $\gamma$-/- orthotopic 
tumors. J. Quantification of CD4 and CD8 T cells in tumors from WT $(n=3)$ and p110 $-/-(n=5)$

mice. Significance testing was performed by unpaired $t$ test. K. Immunofluorescence images of CD8+ T cells in WT and p110 $\gamma-/-$ tumors. Scale bar indicates $50 \mu \mathrm{m}$.

\section{Figure 3: PI3K $\gamma$ inhibition promotes survival of mice with PDAC}

A. Kaplan-Meier plot of survival of $\mathrm{KC}$ mice $(\mathrm{n}=29$; median survival=36 weeks) compared to $\mathrm{KC} ; \mathrm{p} 110 \gamma-/-$ mice $(\mathrm{n}=15$; median survival=43.9 weeks). B. Kaplan-Meier plot of survival of

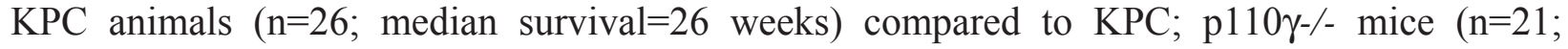
median survival=35.5 weeks). Significance testing was performed by parametric Student's $t$ test. C. Images of hematoxylin and eosin staining and fluorescence immunostaining to detect amylase, claudin and cytokeratin 19 in tissue sections of pancreata tissue harvested at sacrifice in B. Images representative of the group are shown. Scale bar indicates $50 \mu \mathrm{m}$. D. Incidence of invasive carcinoma in animals from B. Significance testing was performed by Fisher's exact test. E. Percent normal acinar tissue in animals from B. F. Incidence of metastasis in animals from B. Significance testing was performed by Fisher's exact test.

Figure 4: Pharmacological inhibitors of PI3K $\gamma$ suppress PDAC growth and metastasis 
A. Merged brightfield and red fluorescent images of mCherry-labeled LMP orthotopic pancreatic tumors from mice that were treated with PI3K $\gamma$ inhibitor TG100-115 or chemically similar control according to the schema depicted. B. Mean area of fluorescence from A. C. Mean weight of pancreata from A. D. Incidence of metastases in animals from A. E. Weight of normal pancreas and TG100-115 and control treated p53 2.1.1 orthotopic tumors. F. Images of tumors from TG100-115 and control treated animals from E. Arrows indicate necrotic tissue. G. Weight of PDAC tumors from animals treated with saline, TG100-115, gemcitabine and gemcitabine+TG100-115 according to the depicted schema. Significance testing was performed by one-sided Anova with Tukey's posthoc multiple pairwise testing. H. Incidence of metastases in animals from G. Significance testing was performed by Fisher's exact test. I. Effect of TG100$115(\mathrm{n}=9$; median survival $=33$ days $)$, gemcitabine $(\mathrm{n}=9$; median survival $=32$ days $)$ and combination gemcitabine + TG100-115 treatment $(n=9$; median survival $=38$ days $)$ on survival of implanted PDAC tumors compared to control treated animals $(n=9$; median survival $=28$ days). J. Kaplan-Meier survival plot of KPC animals that were treated from week 10 to week 25 with TG100-115 $(\mathrm{n}=5$; median survival $=28.5$ weeks $)$ compared to control treated KPC $(\mathrm{n}=5$; median survival $=21$ weeks $)$ and untreated $\mathrm{KPC}$ mice $(\mathrm{n}=11$; median survival $=24.3$ weeks $) . \mathbf{K}$. Incidence of metastasis in TG100-115 $(n=15)$ treated and control treated KPC animals $(n=14)$. Significance testing was performed by Fisher's exact test. L. Images of metastases in liver cross 
sections from control and TG100-115 treated tumors from K. Significance testing was performed by Anova or unpaired t-test.

\section{Figure 5: $\mathrm{PI} 3 \mathrm{~K} \gamma$ drives tumor associated macrophage polarization and immune}

\section{suppression in vitro and in vivo}

A-B. Relative mRNA expression of immune response genes in (A) orthotopic WT and p110 $\gamma$-/-

Panc02 tumors and (B) purified TAM from orthotopic WT and p110y-/- Panc02 tumors. C.

Relative mRNA expression of immune response genes in p110 $\gamma-/-$ or TG100-115 and control treated in vitro macrophages that were polarized with IL-4 for $48 \mathrm{~h}$. ${ }^{*} p<0.05$. D. Mean weight of tumors from WT or p110 $\gamma$-/- mice implanted with p53 2.1.1 tumor cells admixed 1:1 with macrophages isolated from p53 2.1.1 tumors grown $\mathrm{p} 110 \gamma-/-$ and WT mice $(n=8)$. Significance testing was performed by one-way Anova with Tukey's posthoc multiple pairwise testing. E.

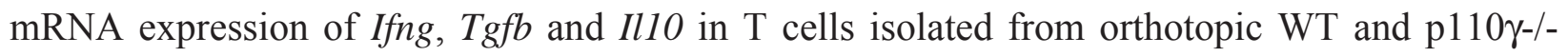
Panc02 tumors. F-G. In vitro proliferation of anti-CD3, anti-CD3+CD28 or IL2 + anti-CD3+ CD28 treated $\mathrm{T}$ cells isolated from spleens of $(\mathbf{F})$ naive mice or $(\mathbf{G})$ p53 2.1.1 PDAC tumor bearing mice. H. p53 2.1.1 tumor growth in WT, p110 $\gamma^{-/-}, \mathrm{CD}^{-/-}$, and p110 $\gamma^{-/-} \mathrm{CD}^{-/-}$animals 
$(n=10)$. Significance testing was performed by one-way Anova with Tukey's posthoc multiple pairwise testing.

Figure 6: PI3K $\gamma$ controls macrophage PDGF expression to promote PDAC tumor cell invasion

A. Chemotaxis of LMP PDAC cells towards conditioned medium from IL-4 stimulated WT or p110 $\gamma$-/- macrophages (IL-4 CM). B. Relative mRNA expression of select chemotactic factors in IL-4 stimulated p110 $\gamma$-/- vs WT macrophages as determined by RNA sequencing and expressed as Log2-fold change from WT. C. Chemotaxis of LMP PDAC cells towards conditioned medium from IL-4 stimulated WT macrophages (WT IL4 CM) or RPMI medium in the presence or absence of dilutions of the PDGFR inhibitor Imatinib. D. Chemotaxis of LMP PDAC cells towards RPMI or conditioned medium from IL-4 stimulated WT macrophages (WT IL4 CM) in the presence or absence of the PDGF inhibitor Fovista. E. Chemotaxis of LMP PDAC cells towards medium (RPMI) or conditioned medium from IL-4 stimulated WT or p110 $\gamma_{-/-}$ macrophages in the presence or absence of $100 \mathrm{ng} / \mathrm{ml} \mathrm{CCL2,} \mathrm{SCF,} \mathrm{PDGF-AA} \mathrm{or} \mathrm{PDGF-BB.} \mathrm{F.}$ Concentration of PDGF-BB in conditioned medium from WT and p110 $\gamma-/-$ basal (mCSFstimulated) and IL-4 stimulated macrophages. G. Concentration of PDGF-BB in lysates from p53 2.1.1 orthotopic pancreatic tumors grown in WT and p110 $\gamma-/-$ mice. H. Relative mRNA 
expression of Pdgfb in tumor-derived CD11b+Gr1- macrophages, CD11b+Gr1lo monocytes,

CD11b+Gr1hi neutrophils and CD11b-Gr1- cells (tumor cells). Significance testing was performed by Anova or parametric Student's $t$ test.

\section{Figure 7: PI3K $\gamma$ promotes macrophage PDGF-BB expression to control PDAC fibrosis}

A. Masson's Trichrome staining of tissue sections of pancreata from WT and p110 $-/-\mathrm{KC}$ and KPC animals. Scale bar, $100 \mu \mathrm{m}$. B. Masson's Trichrome staining of sections of pancreata from control and TG100-115 treated KPC animals. C-D. Images (C) and quantification (D) of picrosirius red staining of sections of pancreata from KPC tumors grown in WT and p110 $\gamma-/-$ animals. Scale bar $100 \mu \mathrm{m}$. E. Collagen I immunostaining of LMP tumors from animals treated with the PI3K $\gamma$ inhibitor TG100-115 or chemically similar inert control. F. Western blot and quantification of collagen I protein expression in LMP tumors from animals treated with the PI3K $\gamma$ inhibitor TG100-115 or chemically similar inert control. G. Relative collagen I mRNA expression in normal pancreata and LMP tumors from animals treated with the PI3K $\gamma$ inhibitor TG100-115 or chemically similar inert control. H. Relative collagen I mRNA expression in primary murine fibroblasts incubated in the presence or absence of stimulus free conditioned medium from IL-4 stimulated WT and p110 $\gamma$-/- macrophages. I. Relative collagen I mRNA 
expression in fibroblasts incubated in the presence or absence of stimulus free conditioned

medium from IL-4 stimulated WT macrophages in the absence or presence of anti-TGF $\beta$, anti-

PDGF-BB or Imatinib. Significance testing was performed by parametric Student's $t$ test. Research. 
A
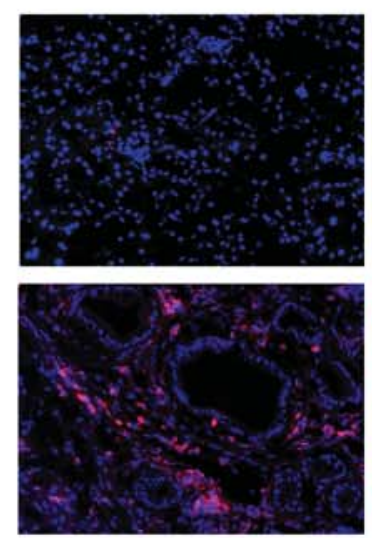

LSL-KRas $^{\text {G12D/+ }}$
Pdx-1Cre

C

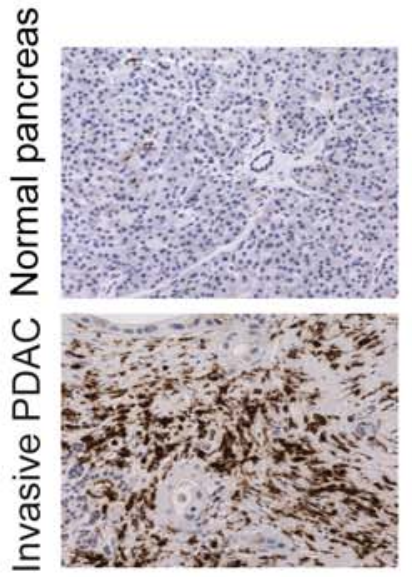

$E$
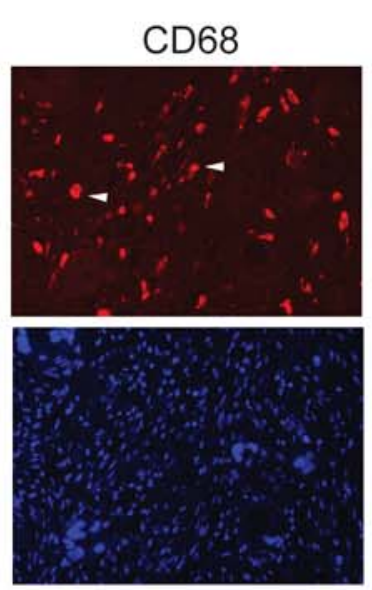

DAPI
Orthotopic
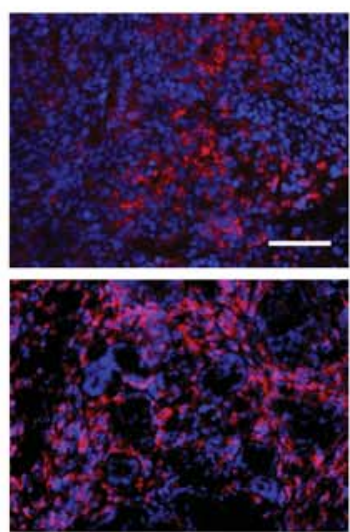

LSL-KRas G12D/+

LSL-Trp53 ${ }^{R 172 H /+}$

$P d x-1 C r e$

B

Figure 1

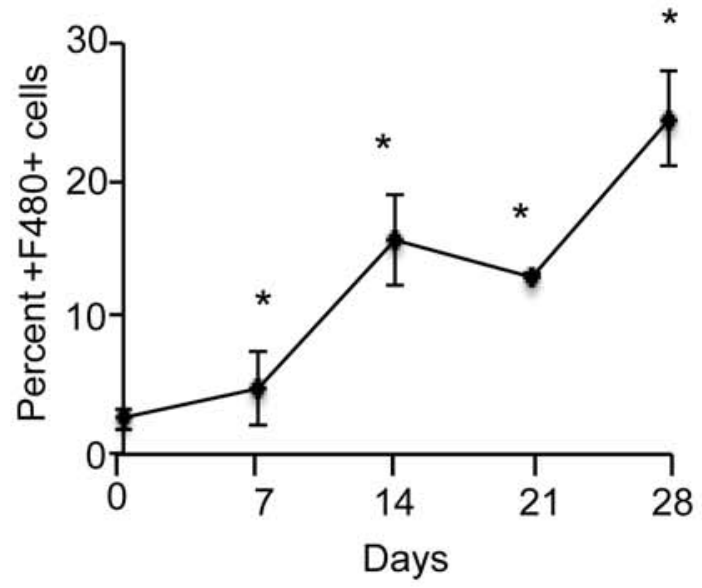

D
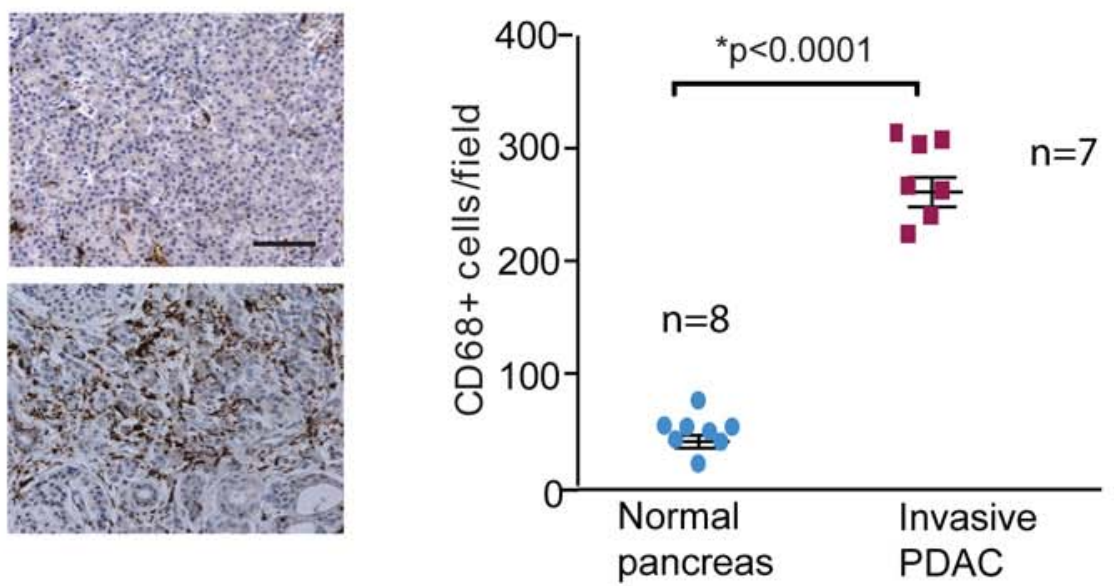

F

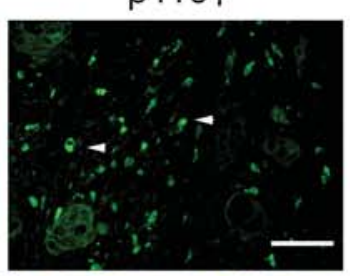

$\mathrm{p} 110 \gamma$

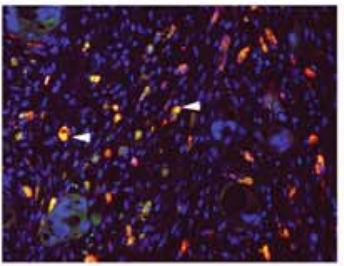

p1108

$\mathrm{p} 110 \alpha$

$\beta$-actin 
A

implant $\downarrow$

Panc02

K8484

p53 2.1.1
B
C

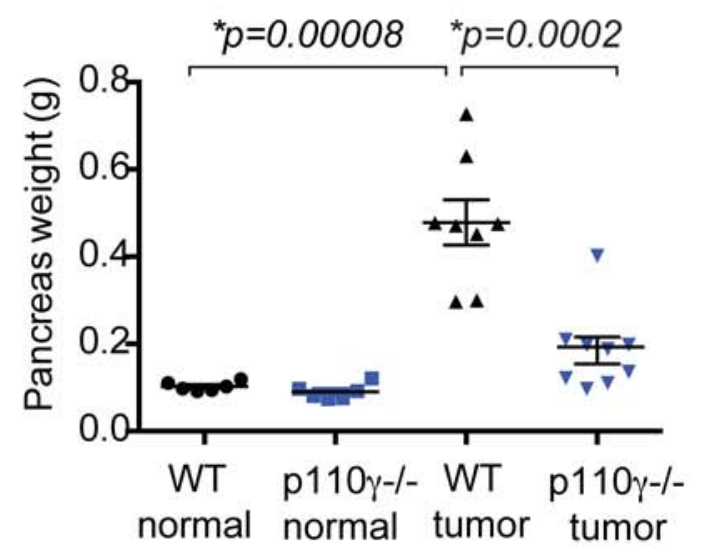

$\mathrm{F}$
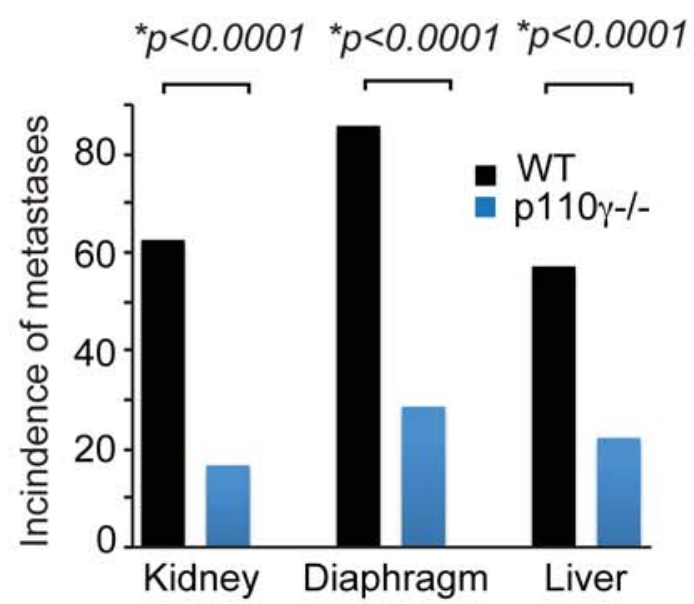

WT $\quad \mathrm{p} 110 \mathrm{\gamma}-/-\mathrm{p} 53$ 2.1.1 M $\Phi \quad \mathrm{M} \Phi$ p110

$\beta$-actin
D

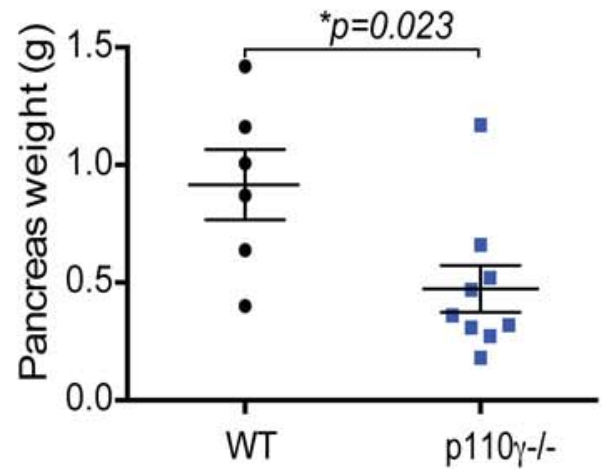

p53 2.1.1

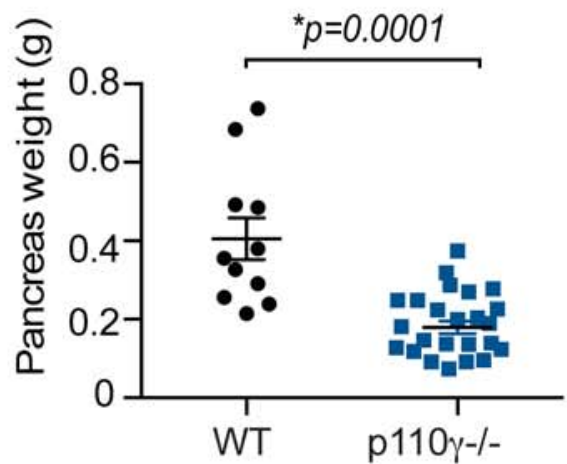

G

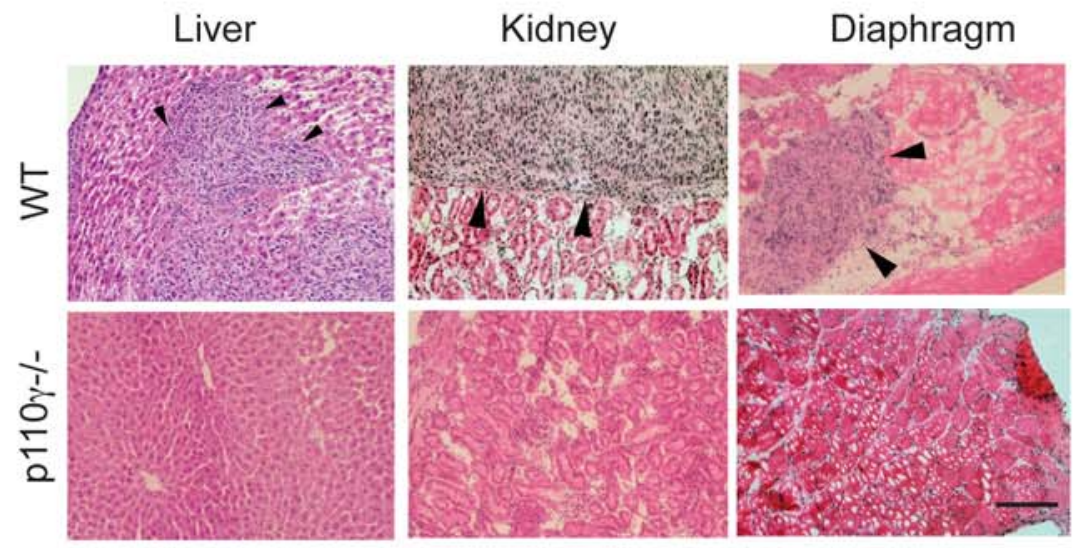

$\mathrm{H}$

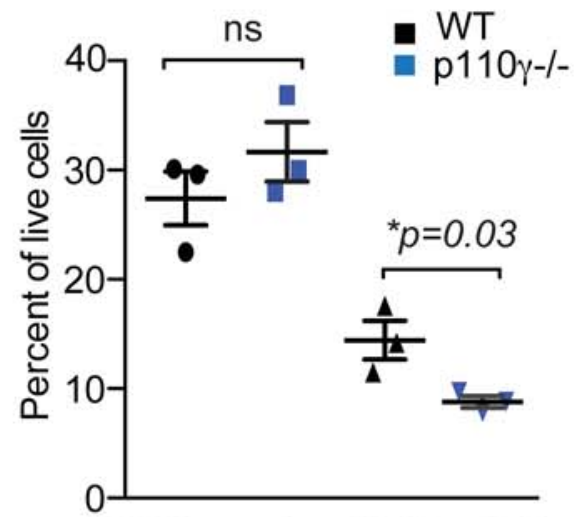

CD11b+Gr1- CD11b+Gr1+ Myeloid cells

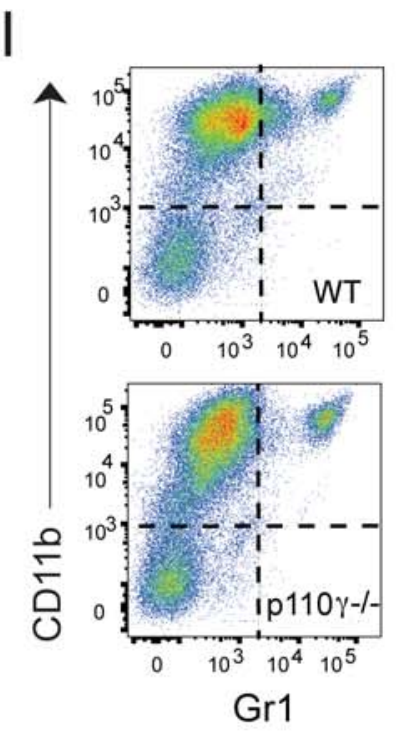

J

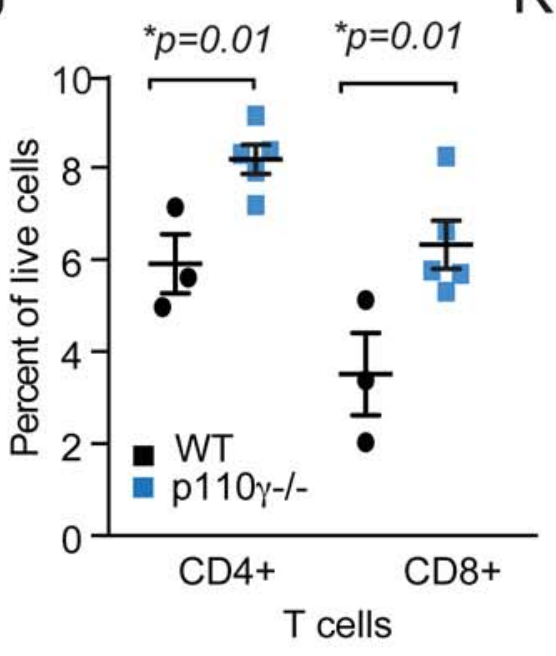

CD8+ $T$ cells

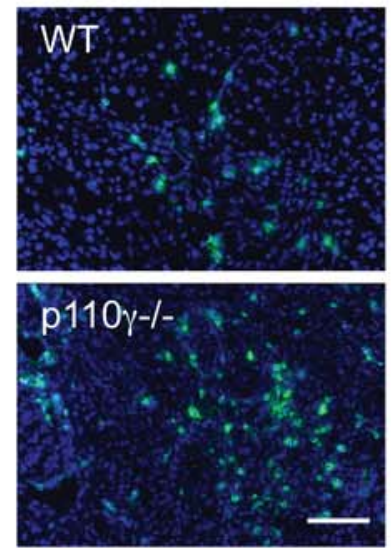



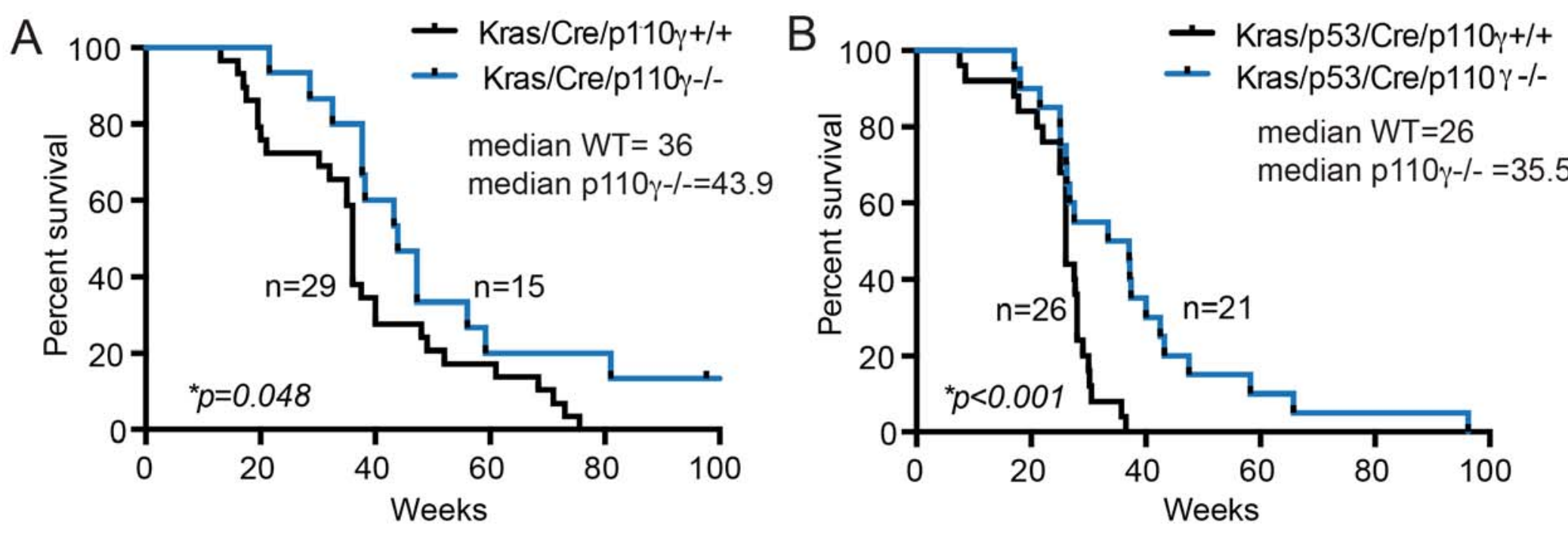

C

H\&E

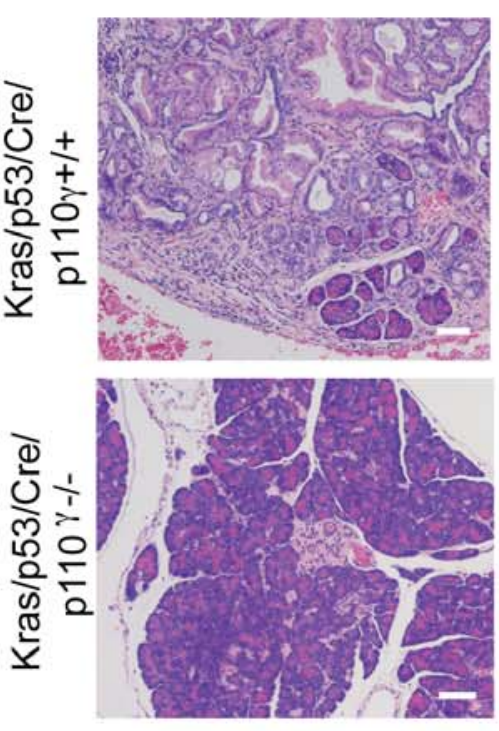

H\&E
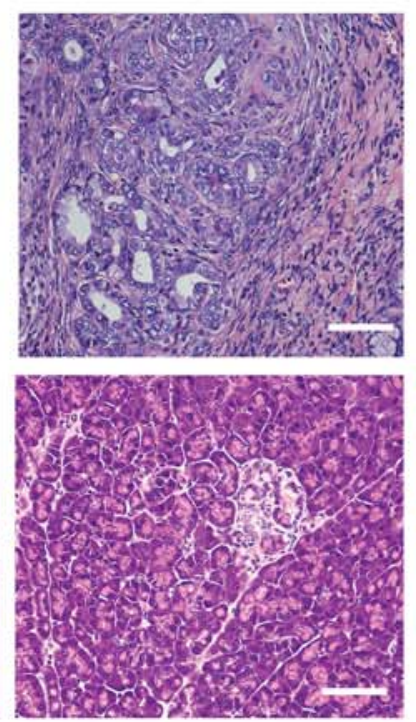

Amylase
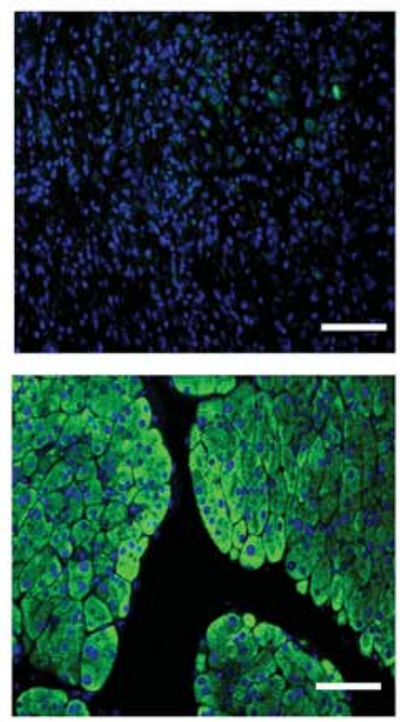

Claudin
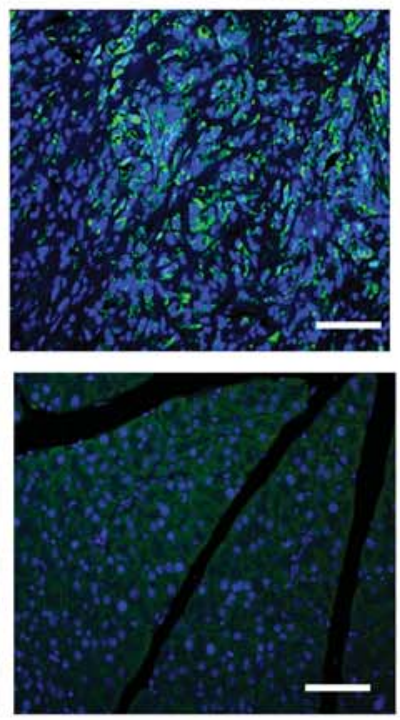

Cytokeratin 19
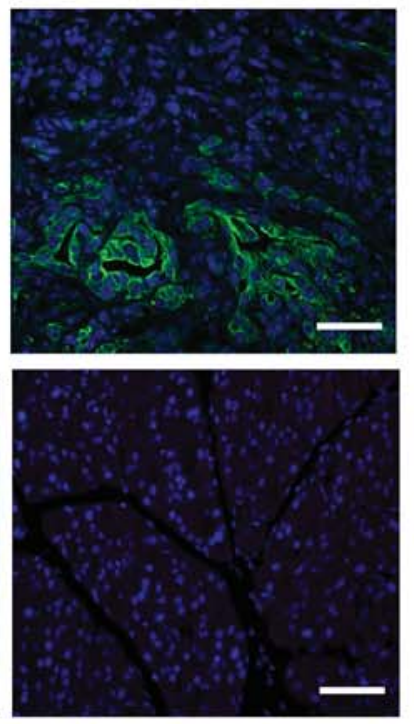

$\mathrm{F}$
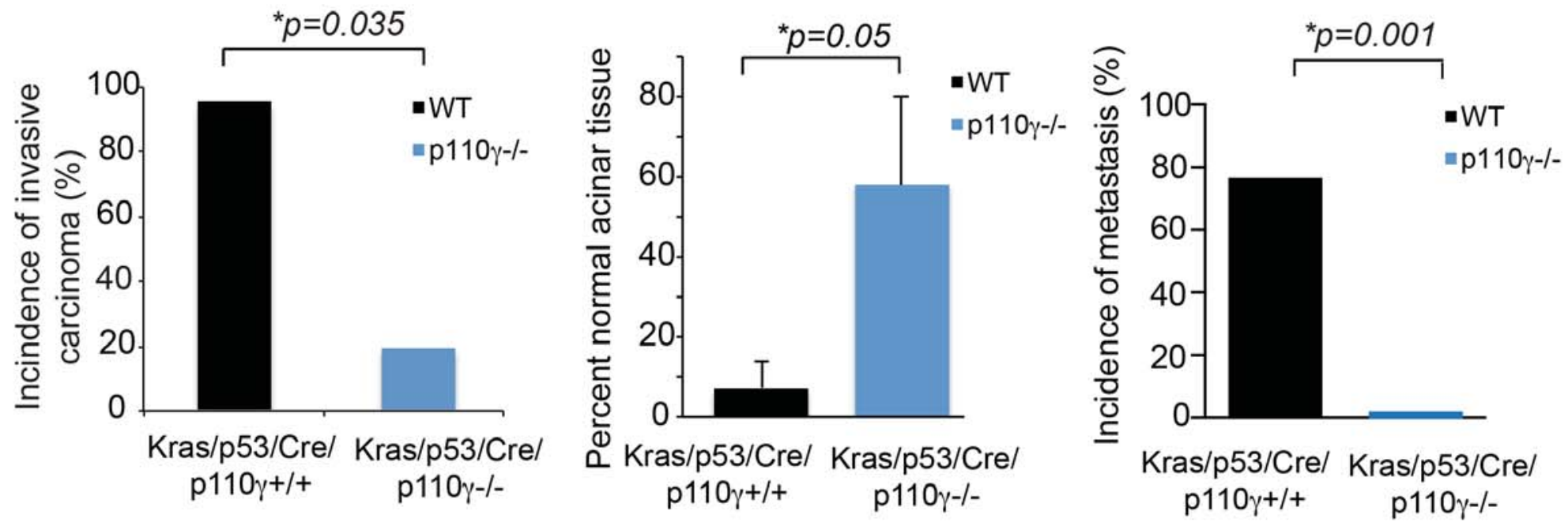
mCherry LMP
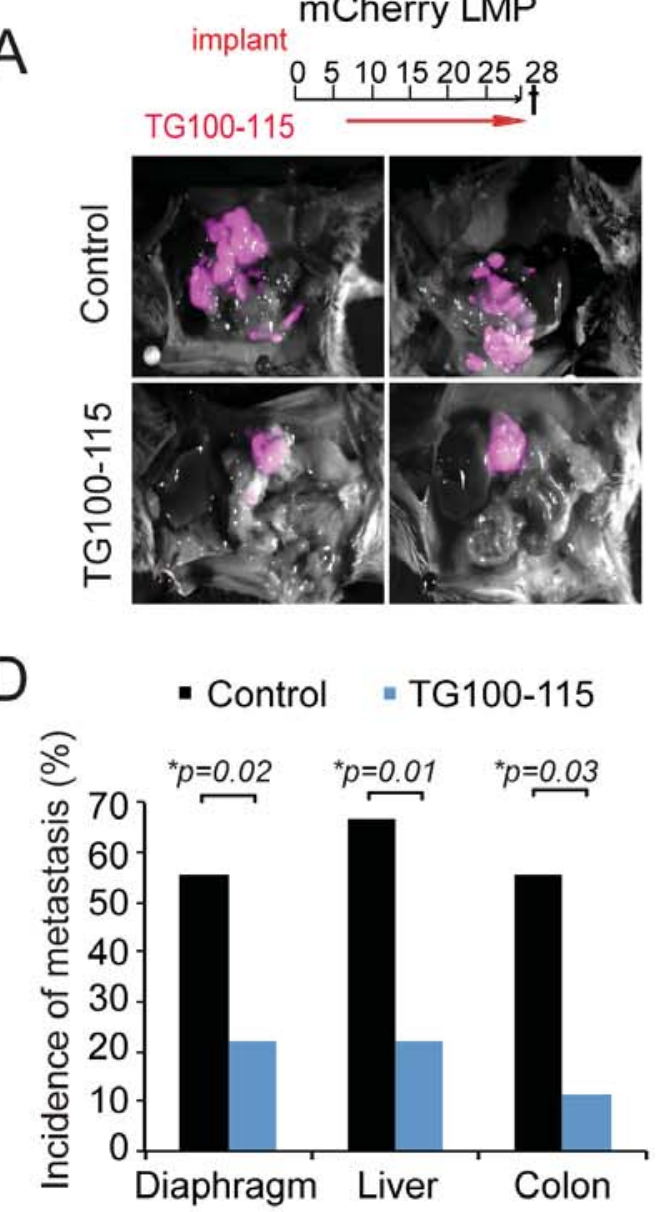

G
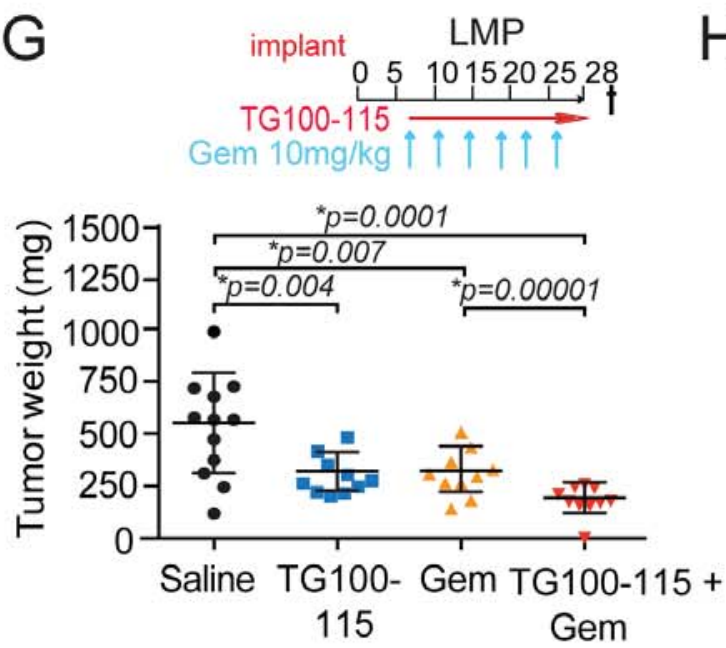

J

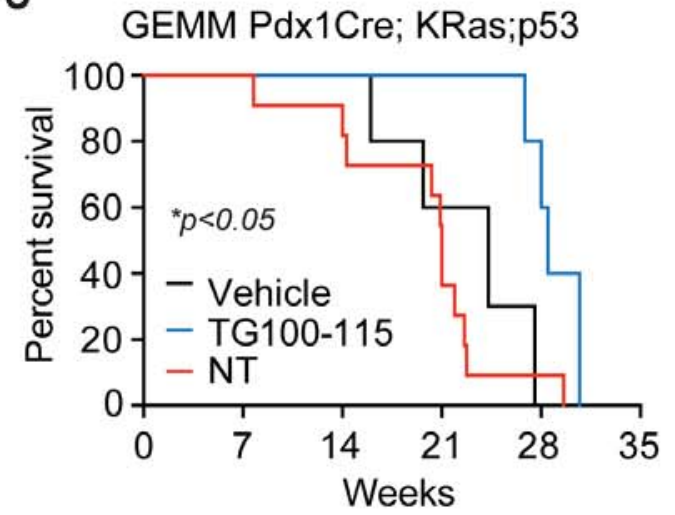

B

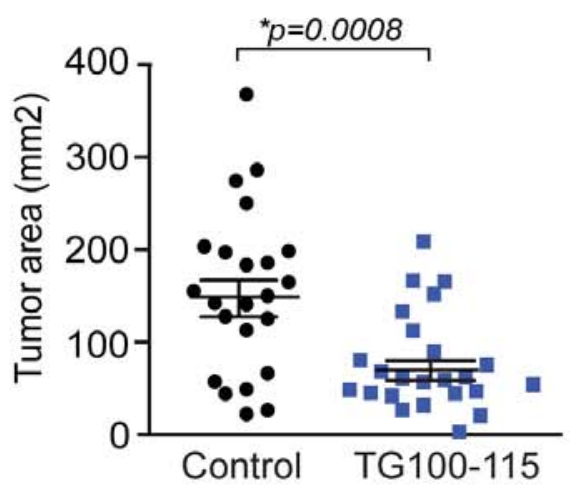

E

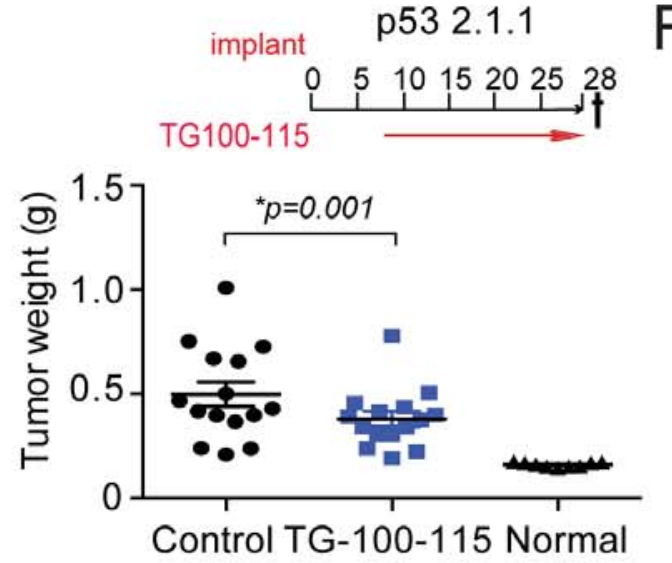

H

Pancreas
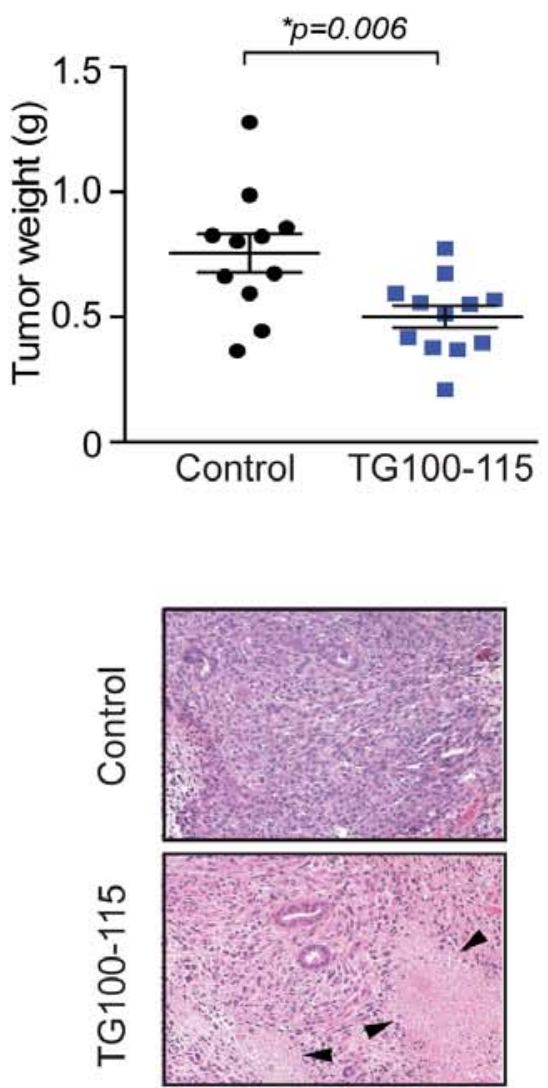

- Saline

- Gemcitabine -TG100-115
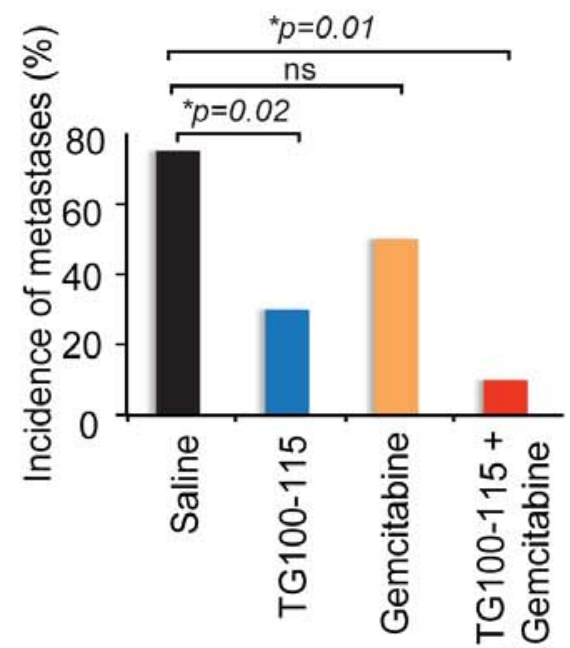

K

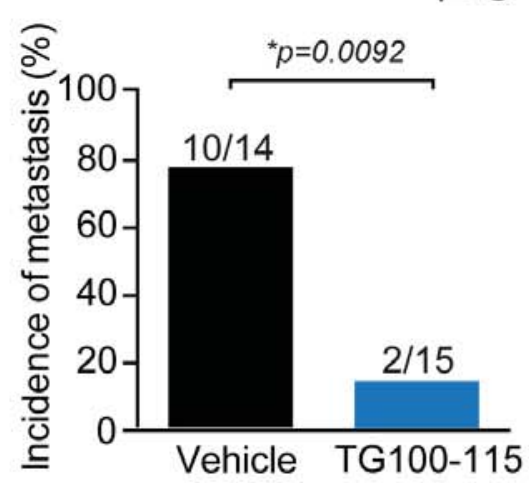

TG100-115 +
Gemcitabine

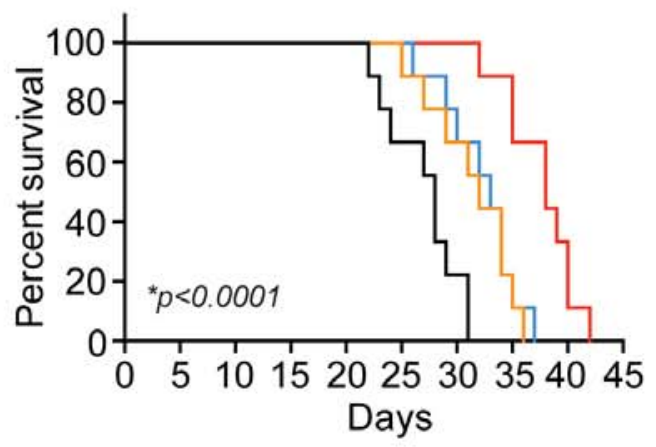


A - wtuthor manuspripts of ave been peer reviewed and ecepted for publication but have not yet been edited. Figure 5
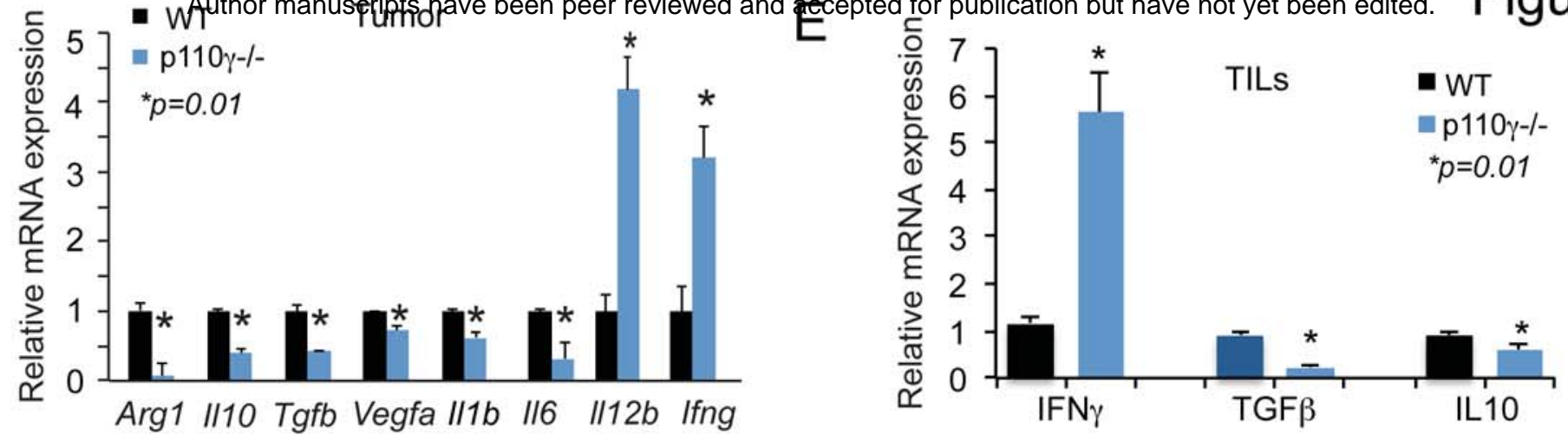

B
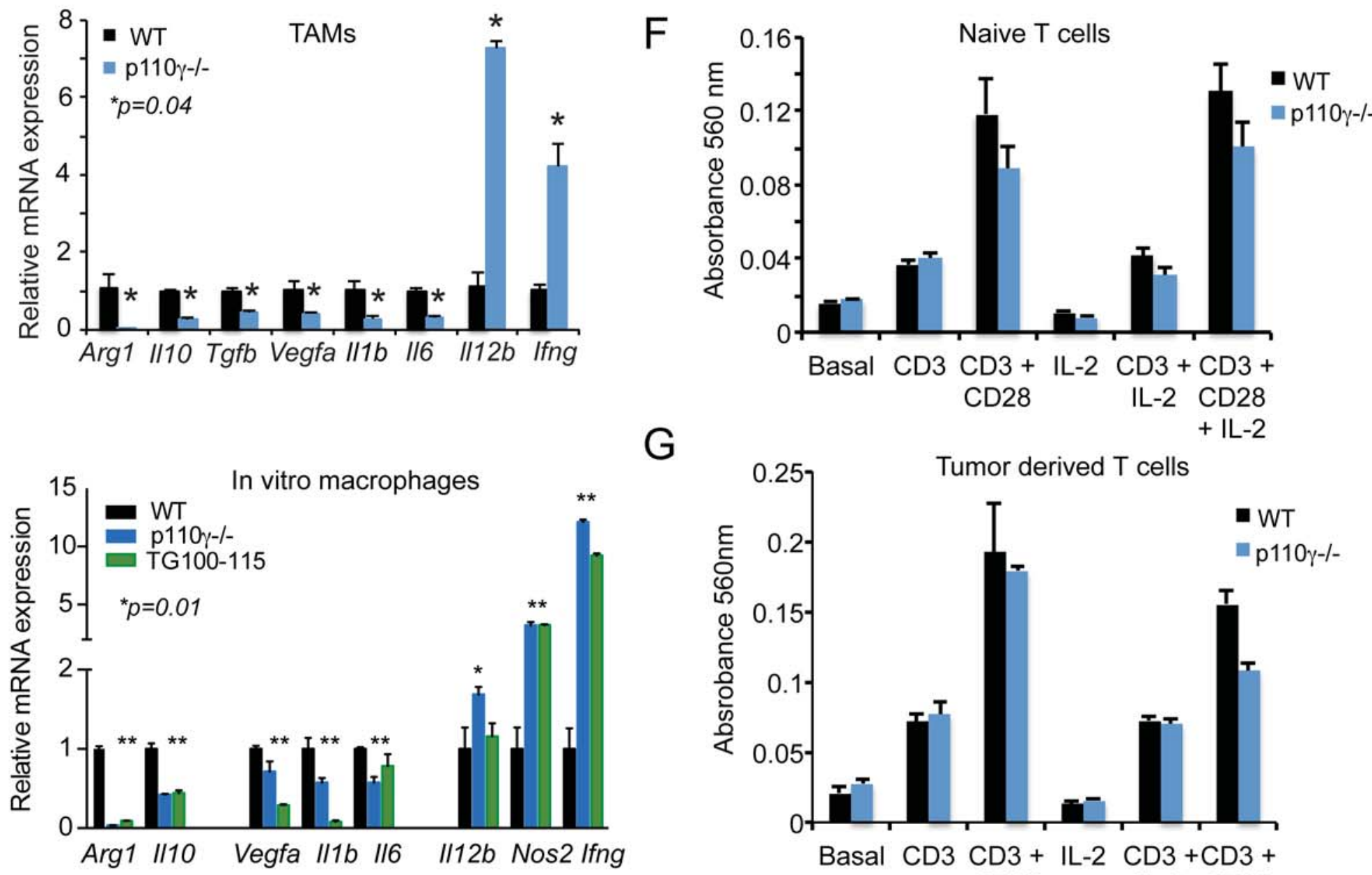

G
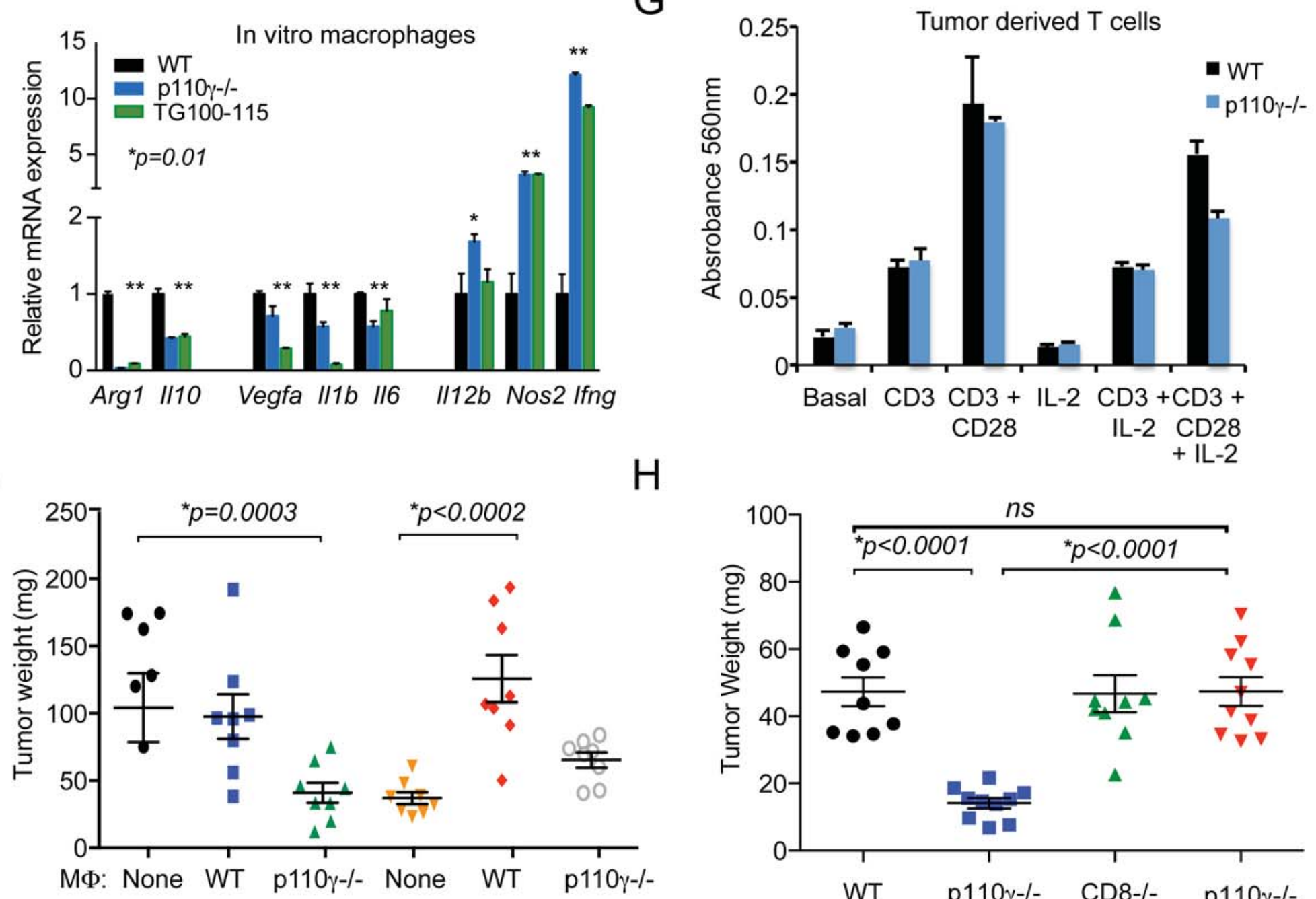

Host:

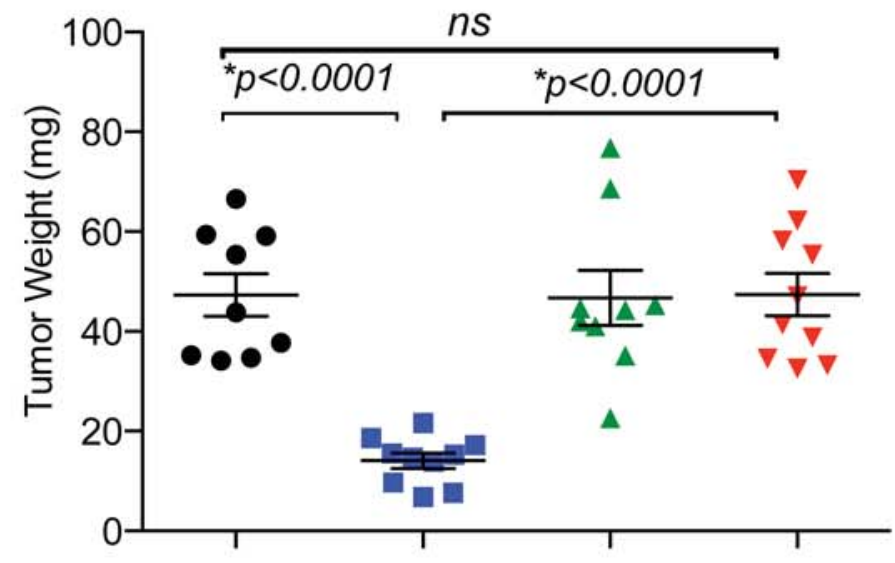

WT

p110 $-1-$ CD8-/-

p110 $\gamma-1-$ Downloaded from cancerdiscovery.aacrjournals.org on June 7, 2016. (c) 2016 American Association for Cancer 
Author Manuscript Published OnlineFirst on May 13, 2016; DOI: 10.1158/2159-829RGP-15-1346

Author manuscripts have been peer reviewed and accepted for publication but have hib yet been edited.

A
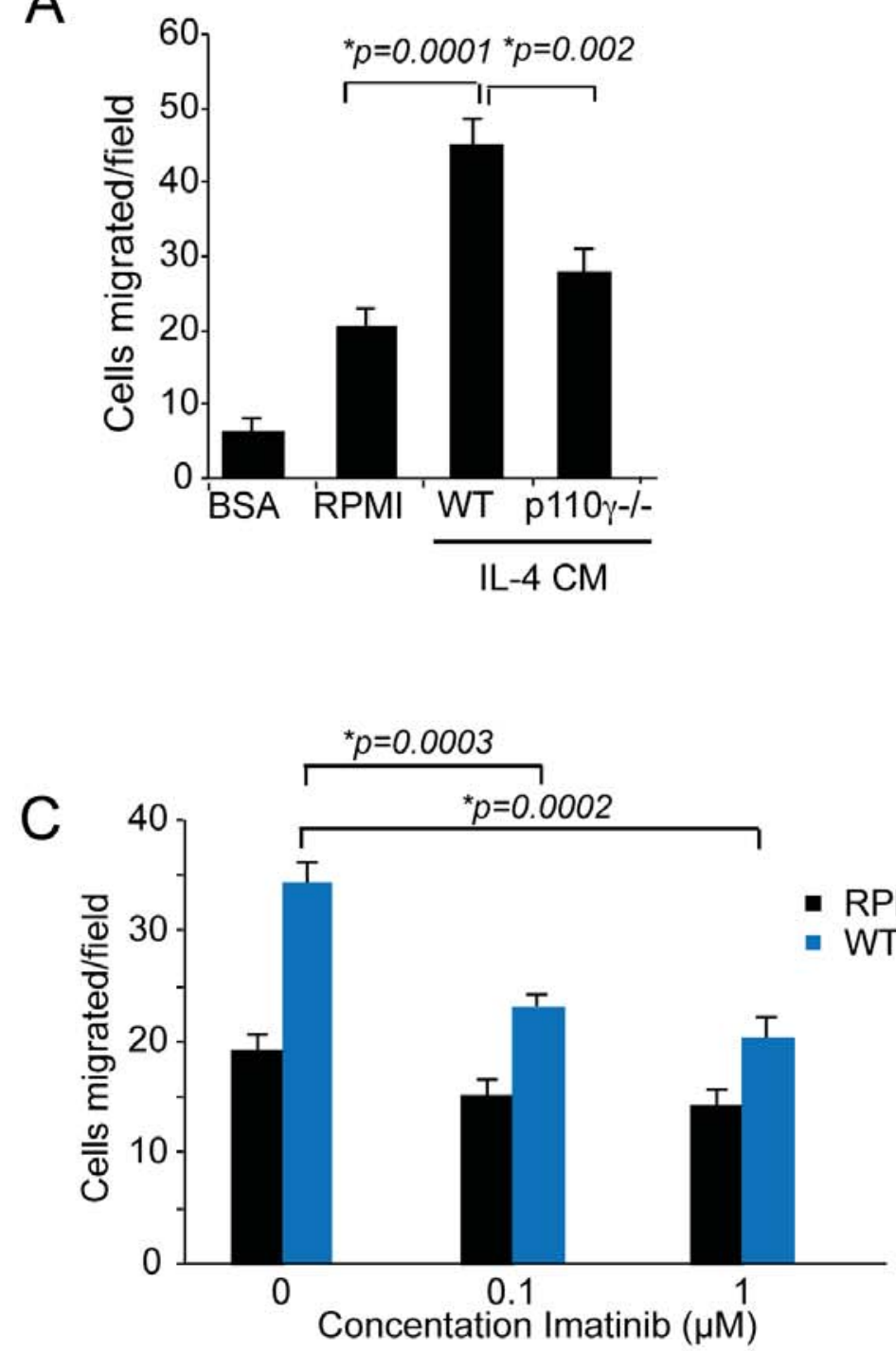

$\mathrm{B}$

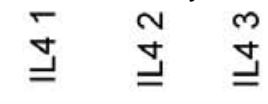

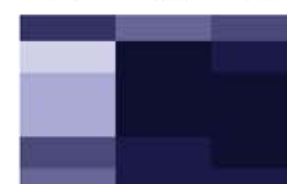

$\mathrm{Pf} 4$
$\mathrm{Ccl} 7$

$\mathrm{CCl} 2$

Plau

II1 rn

Spp1

$\mathrm{Ccl} 4$

$\mathrm{Ccl} 3$

Hbegf

Fgf13

Vegfa

$\mathrm{Pdgfb}$

Mmp10

Pdgfa

F3

Met

Ccl5

Mmp9

Mif

Sema3c

Vash1

Kitl

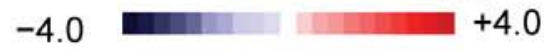

D

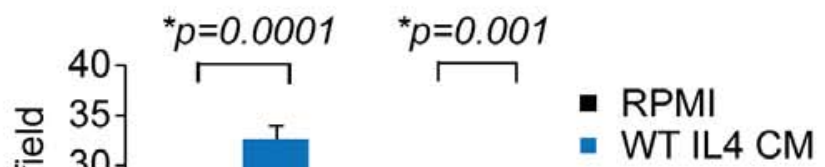

F

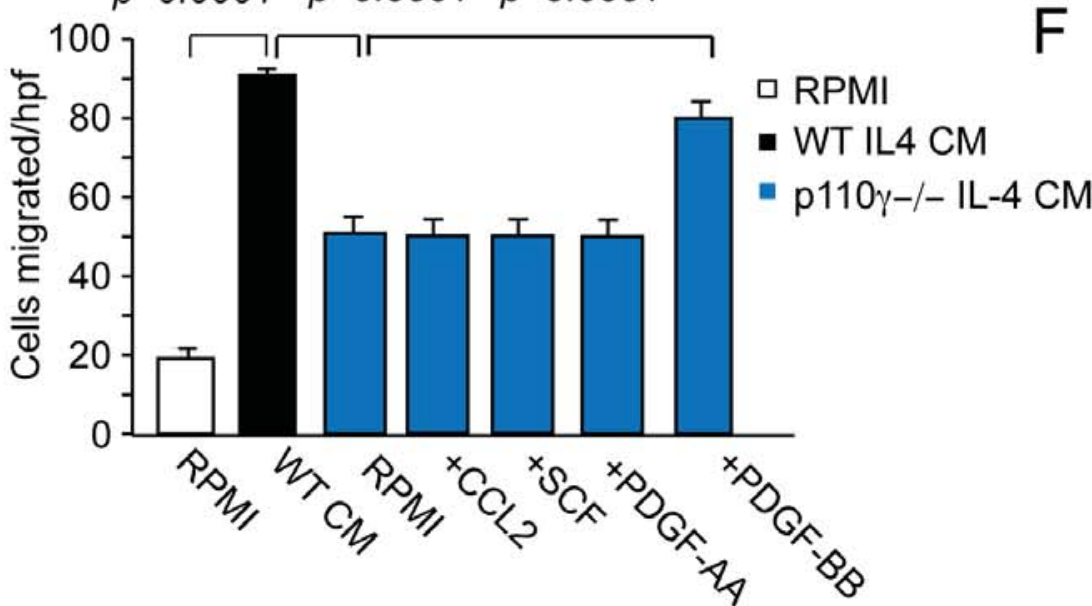

F

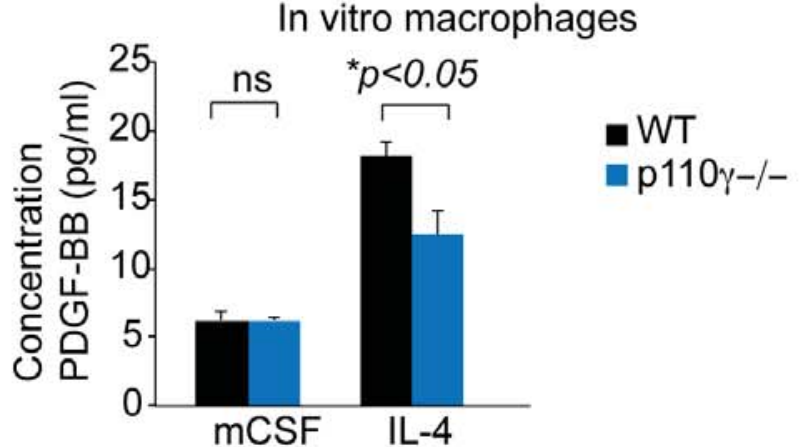

$\mathrm{H}$

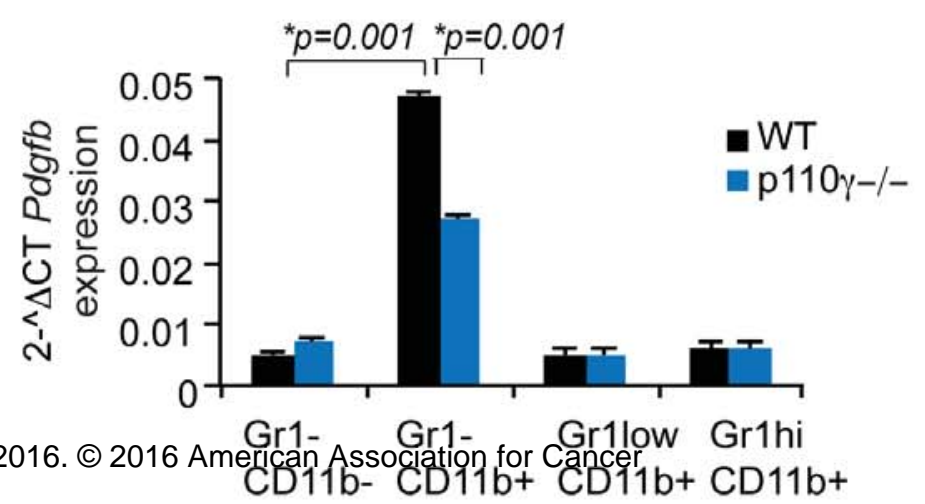


A

Author Manuscript Published OnlineFirst on May 13, 2016; DOI: 10.1158/2159-8290.CD-15-1346

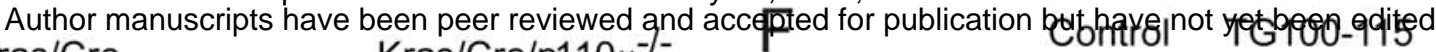

Kras/Cre

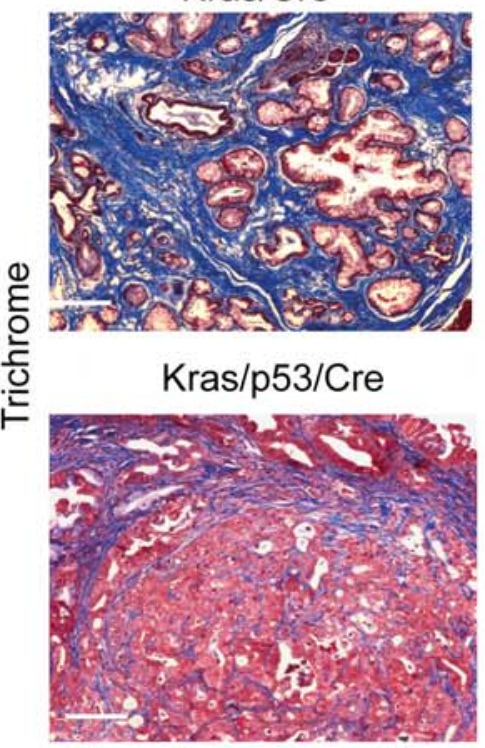

B

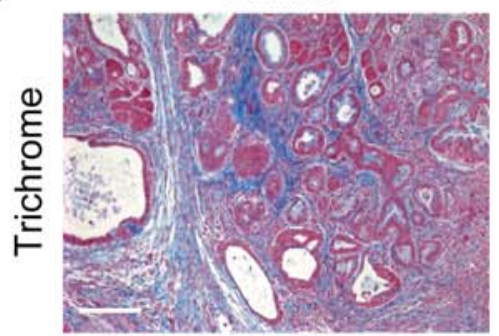

C

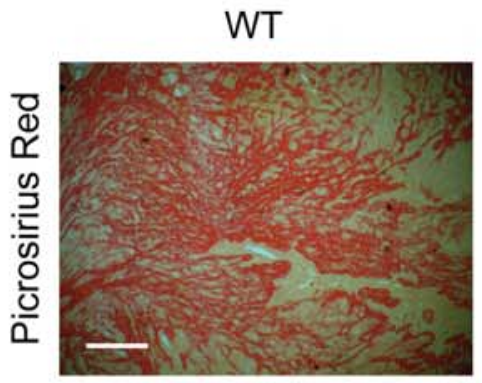
$\mathrm{Kras} / \mathrm{Cre} / \mathrm{p} 110 \gamma^{--}$
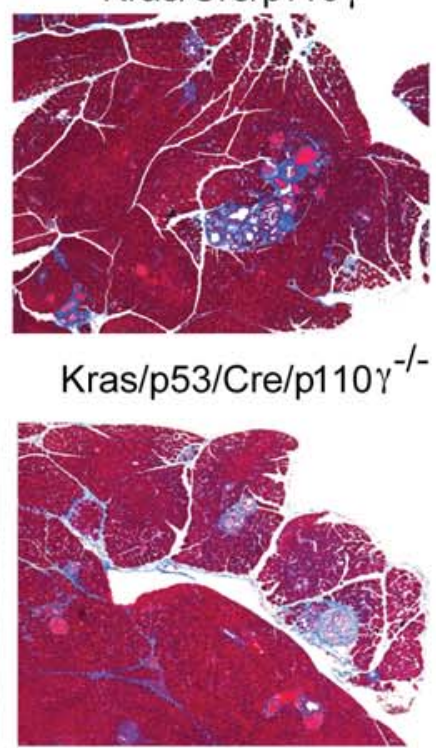

G
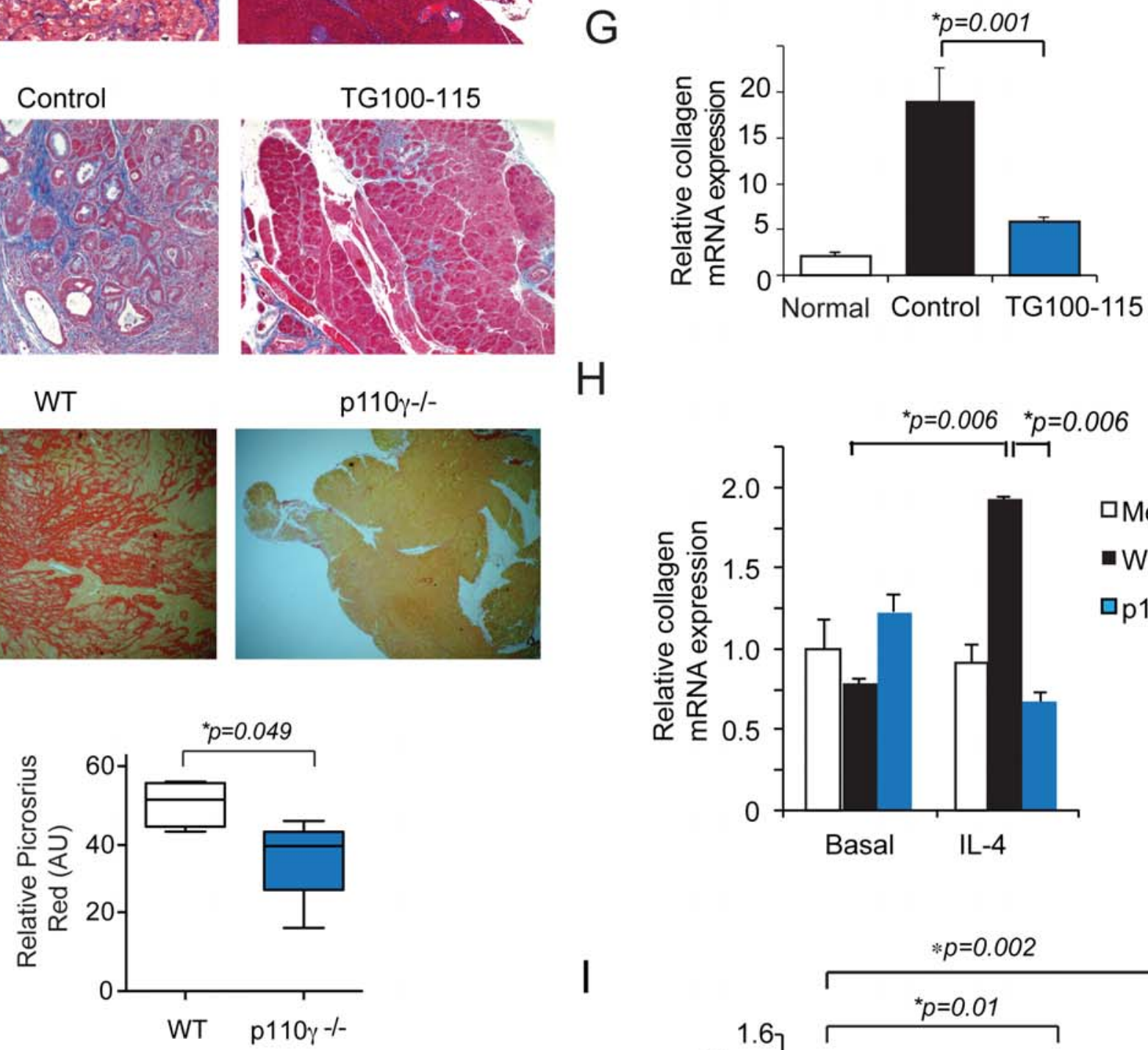

$\mathrm{H}$

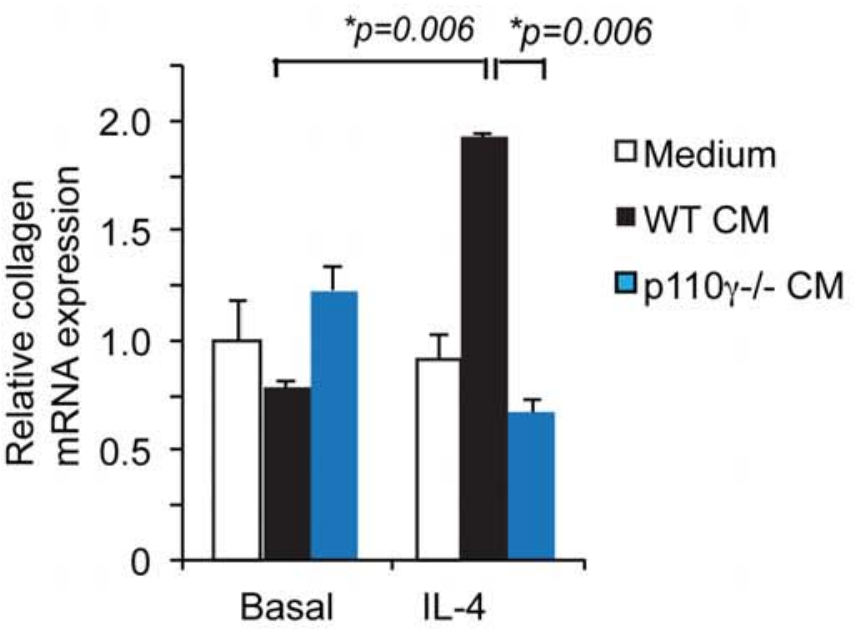

TG100-115

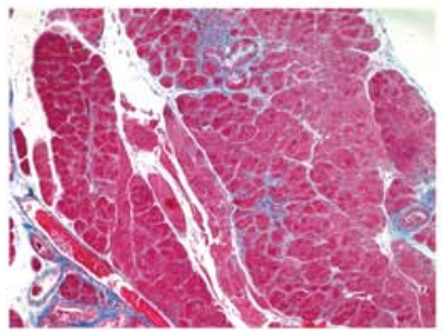

p110 $-/-$

TG100-115

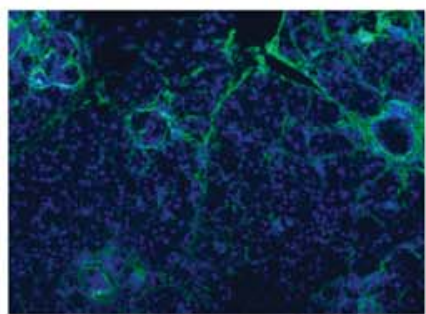

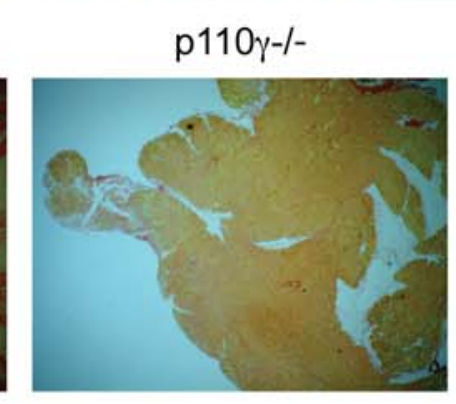

Collagen

Actin
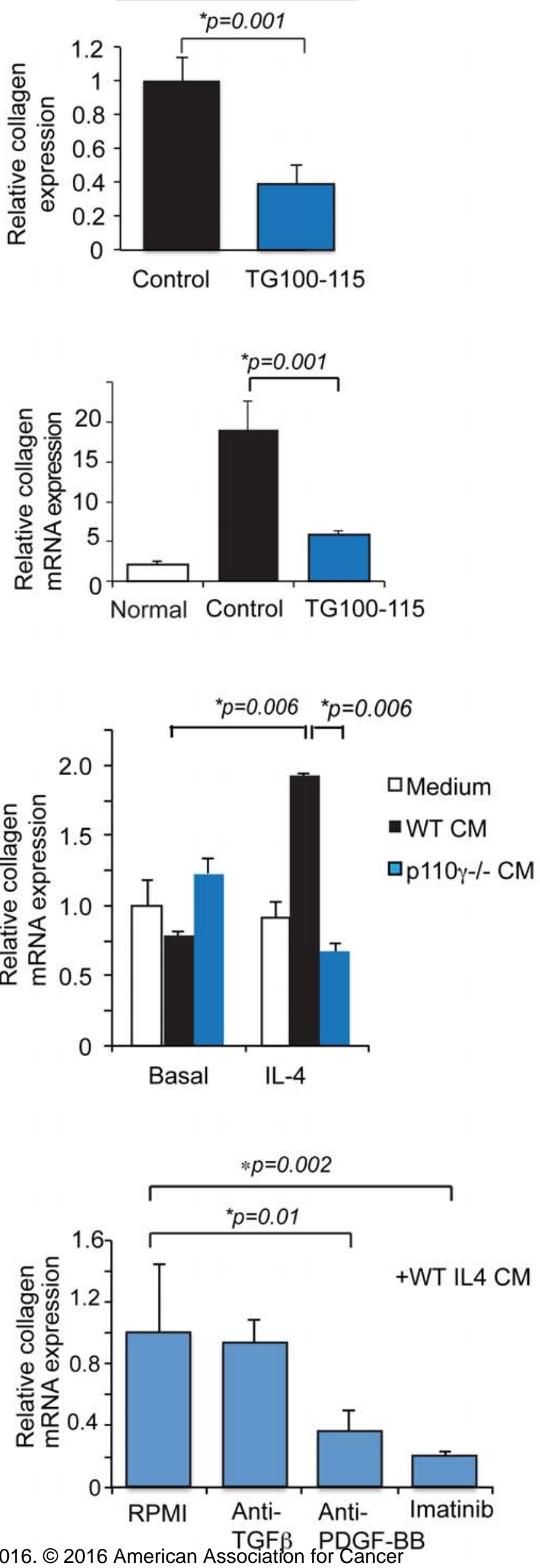

Downloaded from cancerdiscovery.aacrjournals.org on June 7, 2016. () 2016 American Association for Cancer
E
Control

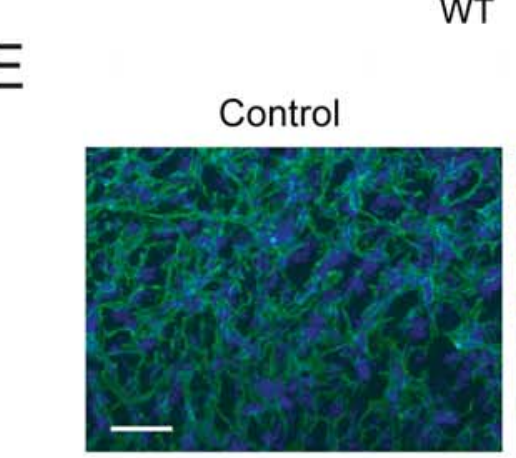

D 


\section{CANCER DISCOVERY

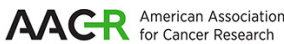

\section{Macrophage PI3K $\gamma$ drives pancreatic ductal adenocarcinoma progression}

Megan M. Kaneda, Paola Cappello, Abraham V. Nguyen, et al.

Cancer Discov Published OnlineFirst May 13, 2016.

Updated version Access the most recent version of this article at: doi:10.1158/2159-8290.CD-15-1346

Supplementary Access the most recent supplemental material at:

Material http://cancerdiscovery.aacrjournals.org/content/suppl/2016/05/13/2159-8290.CD-15-1346.DC1.html

Author Author manuscripts have been peer reviewed and accepted for publication but have not yet been Manuscript edited.

E-mail alerts Sign up to receive free email-alerts related to this article or journal.

Reprints and To order reprints of this article or to subscribe to the journal, contact the AACR Publications Subscriptions Department at pubs@aacr.org.

Permissions To request permission to re-use all or part of this article, contact the AACR Publications Department at permissions@aacr.org. 